\title{
Das Verhältnis der Handschriften des altfranz. Gregorius.
}

Von der altfranzösischen Gregorius-Legende sind uns bis jetzt 5 Handschriften aus dem I2. bis 15 . Jahrh. bekannt.

Die nachstehende Untersuchung soll sich nun mit der Frage beschäftigen, in welcher Weise diese Handschriften mit einander verwandt sind. Den Citaten werde ich im Allgemeinen die Ausgabe Luzarche's zu Grunde legen; nur wo die mit „B“ bezeichneten Handschriften gänzlich von den mit „A“ benannten abweichen, folge ich deren Folionummer und Verszahl.

TEIL I.

\section{CHARAKTERISTIK DER HANDSCHRIFTEN.}

\section{$\S$ I. Die Handschrift von Tours $\left(A_{1}\right)$.}

Die Abfassung der Handschrift $A_{1}$ verdanken wir einem Provenzalen, ihre Entdeckung Victor Luzarche, der in der Einleitung zu seinem Adam, drame anglo-normand du XIIième siècle, Tours 1854 , eine Beschreibung derselben giebt. Ausführlicher handelt hierüber Delisle (Romania II 9i f.).

Die Handschrift befindet sich in der Bibliothèque Communale zu Tours und ist nach dem Urteile des Herausgebers in der ersten Hälfte des I3. Jahrh. in einem Mischdialekte des Loirethals (dialecte ligérien) geschrieben. Sie führt in dem von Dorange herausgegeben Kataloge die Nummer 927. Auf 229 Blättern aus Baumwollenpapier stehen 9 verschiedene Stücke, unter ihnen der von Luzarche herausgegebene Adam. Unsere Legende finden wir auf Fol. 109-185 zwischen „La Vie de Notre Dame“ und „Les Distiques de Caton". Im Jahre 1857 veröffentlichte sie Luzarche unter dem Titel: Vie du pape Grégoire le Grand, légende française, publiée pour la première fois par Victor Luzarche. Tours 1857. Die Herausgabe ist jedoch sehr mangelhaft, und Littré hat im Journal des Savants 1858 , wiederabgedruckt in seiner Histoire de la langue française, Bd. II I $70 \mathrm{f}$., den Text an zahlreichen Stellen berichtigt. Als von Littré nicht berührte, aber auf Schuld des.Herausgebers zu setzende Fehler führe ich noch folgende an: 
berssoïl für berssoil 21, 18 ;

Reim deserté : povreté f. e desérte : povérte 32, 23-24;

querent d̀ le esbaneier f. qu'erent alé esbancier 42, 19;

grammairés es f. grammaire ses 48,3 ;

celes ierent f. s'eles $i$ erent 70, I4;

coursoir f. sours oïr IO9, 20;

le verai f. leverai I 14,7 ;

mist l'a f. mist la I 15 , I 3 ;

bons oions avingement f. bon soions au jugement I I 8, 9;

$e$ i f. chi I 8 I I, wie $\mathrm{B}_{2}$ deutlich liest.

Das Manuskript zeigt eine grölsere Lücke. Es fehlt ein ganzes Blatt, das nach der Ansicht Hugo Bieling's: „Ein Beitrag zur Überlieferung der Gregorlegende, Berlin I 874 p. 23, vielleicht durch die Hand eines mönchischen Fanatikers absichtlich vernichtet worden ist. Um diese Lücke auszufüllen, entlehnte der Herausgeber 64 Verse der Arsenal-Handschrift No. $325\left(B_{2}\right)$, welche sich in seiner Ausgabe p. I09, I I - I I 2,2 inkl., und nicht, wie er in der Einleitung p. XXV angiebt, p. I09,20- I I 2,3 exkl. finden. Demselben Manuskripte entnahm der Herausgeber die 6 Schlufsverse, die nach Zeugnis der mit $A_{2}$ und $A_{3}$ bezeichneten Handschriften als unursprünglich sich erweisen.

Obgleich diese Handschrift den besten Text uns liefert, ist sie nicht frei von mancherlei Versehen, Fehlern und Irrungen. Es würde jedoch zuweit führen, alle diese Verderbnisse hier verzeichnen zu wollen; ich beschränke mich daher auf einige Beispiele.

Fehler sind aus folgenden Gründen in die Handschrift hineingekommen:

I. Buchstaben werden vertauscht.

$e$ f. $a=$ habet $8, \mathrm{I}$;

e f. $a=a d 26,19 ; 44,5 ; 80,3 ; 83,7 ; 97,24 ; 102,7$;

e f. $o=$ apud I 8,$17 ; 64,1 ; 64,8 ; 66,9 ; 115,14 ;$

e f. $o=u b i 20,2 ; 34,14 ; 35,12$;

$e-e$ f. $o-o=a u t-a u t 78,12$;

$a$ f. $e$ 46,2; 74,12;

il f. el 20,6;20,19;30,20;30,21; 114,2;

el f. il 40,23;

a l'en f. ele en 10,2 I;

del f. n'el 12,12;

de f. ne 70,16;

de f. se 71, II; 77,9;

le f. ne 96,24 ; le f. del 56,9;

si f. li 18,$10 ; 56,22$;

fors f. lors 18,24 ;

a dés f. assez $2 \mathrm{I}, \mathrm{I} \mathbf{1}$;

ades f. assez 92,3 ; 
fust f. funt $2 \mathrm{I}, \mathrm{I} 4$;

les f. des 23,22; 61,10;

qui f. lui 29, I 4;

sa f. fait $32,2 \mathrm{I}$;

hues f. nef 36,2 I;

le f. li 39,2 ; 49,9;

el f. es 48,4;

dunt f. tos $54, \mathrm{I}$;

c2 a f. n'i a 54,13;

se f. ne 57,17;

poi el f. par el 58,14;

por fermemenl f. d'or fin en erent, wie ich konjiziere 59,4;

un f. en 59, I I; ont f. out 63.20;

verrement f. serreement 59,18 ;

l'en f. n'en 6o, I7;

l'en f. leur 66,10;

nos f. vos 67,12 ;

vostre f. nostre $36,2 \mathrm{I}$; 106,10;

list f. mist 70, $1 \mathrm{I}$;

vit f. git 73,2 ;

par f. por 67,12;

por f. par 5,8; 79,2 I;

por f. lor IOI,24;

e je serai f. que je ferai $8 \mathrm{I}, \mathrm{I}$;

al marinant f. a lui errant 85,23 ;

sor f. soz 86,14;

antis f. antif 4,4 ;

grief f. gries 92,12 ;

dieu f. bien 92,21;

icel f. a cel 68,15;

je f. ne $103,2 \mathrm{I}$;

de het f. del tot 26,19.

II. Buchstaben sind a usgefallen.

e f. l'a 12,22;

el f. $\operatorname{cel} 32, \mathrm{1} 7 ; a$ f. $a i 48,1$;

$e$ f. en 35,$1 ; 83,2$;

$i$ f. si 41,10 ; ven f. venra 61,22 ;

par tor f. part tort 74,13 ;

demenos f. demenons 80,6 ;

verrez f. verreis 90,22 ;

pot f. plot I 01,7 .

III. Buchstaben werden hinzugesetzt.

li f. i 23,7; fust f. fus 81,9 ; metre f. mete- 39,3 ;

les f. le 29,7; al f. a 44,5;

veneuz f. venuz 57,15 , beeinflufst durch den Reim veuz 57,16 ;

haée f. haé 83,20 , beeinflufst durch den Reim chastee 83,19, welches Wort der Schreiber als chastée auffafste. 
IV. Ähnlich geschriebene oder ähnlich klingende Wörter werden vertauscht.

pechiere, pecheor, pecheors f. peschiere, pescheor, pescheors 37,24; 39, I5; 42,$5 ; 45,10 ; 45,18 ; 45,19 ; 47,6 ; 89,17 ; .91,15$.

mespris f. espris 8,2 ;

conrei f. secrei 55,12 ;

murent f. muerent 108,10;

baiser f. bailler 32,7;

descovrir f. desconfire 37,2 ;

culvert f. cruel $90,1 \mathrm{I}$;

veies $\mathrm{f}$. vies $92, \mathrm{II}$;

feels f. fels 4I,I I;

car f. par 34, I5;

enork f. enuie 42,8 ;

suer f. fuer 49,2 ;

vait f. fait 59, 19;

fait f. veit 60,6;

chatel f. chantel 61,13 ;

chevaliers f. chevals 67,17;

parlement f. payement 84,7 ;

pardon f. prodon 87,16;

soper f. pover 89, I;

tost f. tos $93,2 \mathrm{I}$;

enceintai f. engendrai 78,7 ;

ramente f. ramene 78,24 .

V. Vorhergehende oder nachfolgende Wörter veranlassen, dafs die richtigen verdrängt werden.

je celerai f. jel nurrirai 20,23 wegen des folgenden recelée; a l'ajorner f. ala juer 42, 15 wegen des vorhergehenden matin, das häufig in der Verbindung matin a l'ajorner vorkommt. Die Schreibung Luzarche's ala jorner, und die Erklärung für jorner = passer un jour ist nicht stichhaltig.

VI. Wörter werden aus andern Versen herübergenommen. getent f. querent 25,19 wegen getent 25,2 I; com f. par 3,23 wegen com 3,24; parlerai f. penserai 55, I wegen parler 54,24; biaus f. bel 20,8 aus 20,7 .

VII. Unverstandene Ausdrücke geben Anlafs zu Änderungen.

So deviserent 18,2 . Daher richtiges chose durch terre verdrängt; uns de Raains f. al daarrain 32,3.

VIII. Reime sind fälschlich umgestellt.

aporté : apreste f. apreste : aporte 21,21-22;

esteit : giseit f. giseit : esteit 8,23-24. 
IX. Das Metrum wird gestört: umgekehrt.

I. durch Setzen eines Simplex für ein Compositum oder

trovée f. retrovée 95,23; donc f. adonc 19,22; porte f. emporte 40,2 I; commandement $\mathrm{f}$. mandement 14,7; emplissent f. aemplissent 26,3.

2. durch Wechsel von Synonymen:

premereinement f. premierement 39,2 I; pale f. palie 75,8.

3. durch Vertretung längerer Formen durch kürzere: gaing f. gaaing 19,18 .

4. durch Ausfall von Wörtern:

de vor ce 3,4; se vor descire 6,1 1; e hinter lui 10,2; e hinter pais 32,23; $e$ am Anfange 75,16; $a$ vor dit 25,1 2; $a$ hinter $e$ 27,20; la hinter en 76,17; tel vor marchaant 86,17; ne vor $i$ 93,1 2 .

Neben diesen Auslassungen einzelner Wörter zeigt die Handschrift auch solcher ganzer Verse. Diese Auslassungen lassen sich nun dadurch erklären:

I. dals wegen 2 gleicher oder ähnlicher Wörter in 2 verschiedenen Versen das Auge des Schreibers abgelenkt wurde:

So vermilst man hinter v. 35,8 einen adversativen Satz, der wegen des Ausfalles dreier Verse hinter 35,6 verloren gegangen ist. Die Handschriften $A_{2} A_{3}$ lesen für 35,73 Verse:

$$
\begin{aligned}
& 35,6 \mathrm{a} \text { si fort les coitoit le tormente } \\
& 35,6 \mathrm{~b} \text { qu'il ne metent a el entente } \\
& 35,6 \mathrm{c} \text { fors seul a issir de la mer. }
\end{aligned}
$$

Wegen coiloit in 35,4 und $35,6 \mathrm{a}$ waren $35,6 \mathrm{a}-\mathrm{c}$ ausgefallen, somit aber ein Reim zu 35,8. Aus diesem Grunde schob $A_{1}$ einen Vers in der Gestalt des v. 35,7 ein.

Aus gleichem Grunde fehlen hinter 72,14 2 Verse, und 72,15 ist von $A_{1}$ geändert, weil durch den Ausfall dieser Verse ein Subjekt verloren gegangen ist. Diese Verse 72,1 4a, b und 72,15 lauten in den anderen Handschriften:

$$
\begin{aligned}
& 72,14 a \text { et la dame apres se leva, } \\
& 72,14 \text { b l'uis de la chambre verela, } \\
& 72,15 \text { a la privée chambre ala. }
\end{aligned}
$$

Wegen chambre in 72,14 und 72,14b fielen $72,14 a$ und $b$ aus.

Eine gleiche Lücke ist hinter 108,16 , die $\mathrm{A}_{2} \mathrm{~A}_{3} \mathrm{~B}_{2}$ nicht haben. Sie lesen:

108,16a or ne volent plus demorer
$108,16 \mathrm{~b}$ icil qui l'en doivent mener.

Sie fehlen vielleicht wegen doivent $108,16 \mathrm{~b}$ und donent 108,18 , 
II. dals gleiche oder ähnliche Versausgänge das Auge des Schreibers von der richtigen Zeile abzogen:

So beweist das car in 42,13, da/s Verse ausgefallen sein müssen. Die anderen Handschriften zeigen auch einen Einschub zwischen 42, I I-I 2. Da hier $A_{3}$ das Ursprüngliche $z u$ haben scheint, citiere ich nach dieser Handschrift:

$$
\begin{aligned}
& \text { 42,I Ia Puis ne fut pas cel plait celez, } \\
& 42,1 \text { Ib que a petit de mal talent } \\
& 42,1 \text { Ic luy reproucha moult laidement } \\
& 42,1 \text { Id la dame qu'il estoit trouvés, } \\
& 42,12 \text { et qu'il n'esfoit du pays nés. }
\end{aligned}
$$

Wegen trouvés in 42, I I und 42,IId fielen alle dazwischen liegenden Verse und 42, I Id selbst aus.

Aus gleichem Grunde ist wohl hinter 101,5 eine Lücke gekommen, die schon Bieling bemerkte. Zwei Gründe sprechen für die Annahme einer Lücke. Erstens fehlt zu apareilerent ein Objekt, das in verloren gegangenen Versen zu suchen ist, oder wenigstens müfste ein se vor apareilerent treten, da dieses Verbum keine reflexive Bedeutung hat. Zweitens kann v. IO I,6 nicht ursprünglich sein, da querent mit apareilerent unmöglich ursprünglich gereimt worden ist. $A_{2} A_{3}$ haben denn auch für 101,65 andere Verse. lch citiere nach $\mathrm{A}_{3}$ :

$$
\begin{aligned}
& \text { 101,5a clercs qui pour luy y envoierent } \\
& \text { 101,5b o grant avoir que ilz porterent } \\
& \text { 101,5c par mains pays querre l'alerent } \\
& \text { 101,5d Partot que ilz oncques aloient, } \\
& \text { 101,5e ce qu'ilz queroient, demandoient }
\end{aligned}
$$

Die Verse $5 \mathrm{~d}$ und e sind wohl Einschub von $\mathrm{A}_{2} \mathrm{~A}_{3}$, da sie tautologisch zu 5c sind. Ist diese Annahme richtig, so sieht man leicht, dafs der Blick des Schreibers wegen der 4 gleichen Reimendungen von apareilerent in 5 auf l'alerent in $5 \mathrm{c}$ fiel, welcher Vers später in die Gestalt des Verses 10 I,6 geändert wurde.

Ohne erkennbaren Grund fehlen in $A_{1}$ hinter 79,18 2 Verse, die sonst lauten:

$$
\begin{aligned}
& 79,18 \text { a mais or ay puis tant pechié fait } \\
& 79,18 b \text { s'il me devoit estre retrait. }
\end{aligned}
$$

Neben diesen Auslassungen zeigt nun aber auch $A_{t}$ nicht ursprüngliche Zusätze und Erweiterungen. So die Verse 52,17-20, die eine unerträgliche Tautologie zu den Versen 52,14-16 sind; ferner die Verse 57,9-10, welche unnötig sind wegen der Verse 56,14 und 56,17-18; endlich auch wohl die Verse 26,9-10, die tautologisch zu 26,8 sind. Vielleicht steht freilich, durch tonel 26,8 beeinflufst, tonelet für bersolet; doch selbst in diesem Falle scheinen sie Einschub zu sein, da sie wegen ihres ungeschickten Satzgefüges kaum aus der Feder des Dichters geflossen sein können. 
Endlich erweisen sich als Fehler der Handschrift: Umstellungen.

So die Verse 3,9-10 und 50,21-22. Letztere müssen vor 50, I9-20 stehen, da sonst die Konstruktion gestört wird. Der Sinn wird entstellt durch Vorschieben der Verse 38,15-16 vor 38,17-20, denn nicht den reichen, sondern den armen Fischer hat der Abt schwören lassen, dafs er darüber schweigen solle, auf welche Weise das Kind gefunden worden sei. Dies ergiebt sich aus den Versen 45,23-46,2.

\section{§ 2. Die Arsenalhandschrift No. $283\left(\mathbf{A}_{2}\right)$.}

Die Handschrift $A_{2}$ bildet einen Teil der unter dem Titel "Légendes des Saints en vers" bekannten und berühmten Sammelhandschrift der Arsenalbibliothek zu Paris Belles-Lettres françaises No. 283 .

Beschrieben ist sie in Francisque Michel's Lai d'Ignaurès, Paris I 832 p. 35 ff., in dem Essai sur les fables indiennes et sur leur introduction en Europe par A. Loiseleur Deslongchamps, Paris 1838 p. XXXIV ff., und in den Mélanges d'archéologie par Cahier et Martin I 93, wonach sie in Beauvais abgefalst ist. Sie ist ein Pergament in fahlrotem Kalbslederband mit vielen, zum Teil auf Goldgrund stehenden Miniaturen. Eine grofse Anzahl derselben ist jedoch herausgeschnitten. So auch die vor unserer Legende. Geschrieben im Jahre I 267 oder I 268 zeigte die Handschrift ursprünglich 365 Folioseiten. Jetzt indessen fehlen 14 Blätter. Die Folioseiten haben 3 oder 4 Kolumnen, deren jede gewöhnlich 50 Zeilen enthält. Titel und Kapitel sind durch rote Buchstaben angezeigt. Am Anfange des Kodex steht ein Index der einzelnen Stücke und deren Folios. Unsere Legende mit der Überschrift: Or orres de saint Gregoire, füllt Fol. $106^{\mathbf{a}}-112^{\mathrm{b}}$. Der Dialekt ist der pikardische.

Verdorben ist der Text durch den Schreiber dieser Handschrift:

I. weil er sich allerlei Schreibfehler hat zu Schulden kommen lassen:

la f. li 6,6; le f. li 13,14 ; li f. le 15,24 ; les f. ses 27,15 ; orroient f. octroient 21,14 ; qu'il f. qu'el 28,4; k'uns f. li uns 38,5 ; contee f. contree 38,10 ; pecheor f. pescheor 45,19; fust f. fist 55, 17; velt f. veit 80,17; l'ame f. dame 81,2 1 ; ot f. or 85,15; k'ai f. n'ai 89,9 ; corage f. orage $94, \mathrm{I}$; bien dist f. brandist 62,6 ; on f. onques 18 , 14 .

II. weil er Wörter aus anderen Versen herübergenommen hat: 
par f. cest 3,24 wegen par 3,23; rien am Anfange zugesetzt I 3, I wegen des Schluls -rien 1 2,24; estre f. muer 2 I, I 6 aus 2 I, I 5; oire apareillier f. oire avancier 30,4 aus 29,24 ; bonte $\mathrm{f}$. charité $89, \mathrm{I} 4$ aus 89,13 ; sept pies f. deus pies 104,8 in Anschlufs an sept ans 104,3; onor f. amor II 6,2 aus I I6,I.

III. weil er durch selbständige Änderungen Tautologien $z u$ früheren Versen schafft:

anchois se fist amer a tous 4I, I I f. il ne fus pas fels ni estous, tautologisch $\mathrm{zu} 4 \mathrm{I}, \mathrm{I} 3$ amer se fist sor tote rien.

IV. weil er Reime fälschlich umstellt:

norir : morir f. morir : norir 25,5-6.

V. weil er durch selbständige Änderungen die Reime stört:

noise: acoilé f. noise: acoile 9,9-10 wegen Änderung des v9, I $\mathrm{o} \mathrm{zu}$ : quant ses beaus frere l'ot acoilé;

moilliers : herbergiez f. moillier: herbergier 87,2 I-22 wegen Änderung des v. 87,22 zu:

que il sempres fu herbergiez;

pris : dist f. pris : quis 36, I5- I6 wegen Änderung des v. 36,16 zu: li uns respondi et dist.

VI. so dals er das Metrum verletzt:

I. durch Setzes eines Kompositums für ein Simplex: raconta f. conta 86,6 .

2. durch Ersatz kürzerer Formen durch längere: desesperance f. desperance 3,5.

3. durch Auslassung von Wörtern:

le vor gart 6,17; ad vor aise 8,5; nul vor confort 21,8; que hinter por ce 35,5; et hinter lire 41,16.

An diese Auslassungen einzelner Wörter schliefsen sich die Auslassungen ganzer Verse.

Einzelne Verse können nur durch Unachtsamkeit und mit Reimstörung ausgefallen sein. So v. 64,24.

Verspaare können ausgefallen sein:

I. wegen gleichen oder ähnlichen Versanfanges.

I I,I7- I 8 wegen des versanlautenden quant in $\mathrm{I} I, \mathrm{I} 7$ und 11,$19 ; 62,15-16$ wegen bien in 62,15 und $17 ; 65,3-4$ wegen d'icel 65,3 und de cels 65,$5 ; 84,1-2$ wegen les nuz vestir 83,24 und 84,$2 ; 96,2 \mathrm{I}-24$ wegen toz $96,2 \mathrm{I}$ und 97,$2 ; 99,5-6$ wegen que 99,4 und 99,6 ; I I I, I 9-20 wegen et I I I, I 8 und I I I,20; I I 4, I 6 und I I 4, I 8 (v. I 6 wegen jor I 5 und I6; v. I 8 wegen der Versschlüsse vie und enuie 17 und I8); 20,23-24 wegen que 22 und 24, 
II. wegen gleichen oder ähnlichen Versschlusses:

$53, \mathrm{I}$ I-I 2 wegen talent I 2 und $14 ; 60,3-4$ wegen descort 4 und desconforter $6 ; 62,7-8$ wegen entrefierent 10 und entredoner 8; 7 I,8-I I wegen segnor 7 und I I; I I 4,9-10 wegen der 4 Reime auf -ens 9-I 2, vielleicht auch wegen tens 10 und sens I 2 ; I 15,9 bis Io wegen der 4 Reime auf -ent 7-10; 23,5-6 wegen petis 6 und petit 8; 21,23-22,4. Durch Umstellung sind nämlich 22,5-6 hinter 24,13-I 4 gekommen. Letztere zeigen aber den Reim duel : bercuel. Hinter diesen hatte wohl eine $\mathrm{A}_{2}$-Handschrift die Verse 21,23-22,24, die aber ausfielen, weil die Verse 22,3-4 auch den Reim bercuel : duel hatten.

III. wegen gleicher oder ähnlicher Wörter im Innern verschiedener Verse:

$37,2 \mathrm{I}-22$ wegen mars 20 und $22 ; 45,7-14$ wegen dist l'abes in 45,7 und 45, 1 5. (Der Schreiber hatte v. 7 mit filleul angefangen. Von dem dist l'abes in v. 7 fiel aber der Blick auf d'ist l'abes in v. I 5. So kam hinter den Anfang des v. 7 der Schlufs des v. I5). Ferner 81,18-20 durch I Vers vertreten. Wegen conois in 17 und 19 war wohl 19 und 20 ausgefallen, wodurch später v. I 8 geändert werden mufste $\mathrm{zu}$ :

que ja conoistre nel quesisse

Dann $82,5-8$ wegen mesfait in 4 und meffais in 8 gemäls der Lesart von $\mathrm{A}_{3}$ :

selonc nos meffais le guierdon.

$84,7-8$ wegen bien und $m a l$ in 8 und 10; 115,4 und 115,6 . Ersterer Vers wegen ferai und verai in 4 und 3 , der andere wegen de toi, das $\mathrm{A}_{2}$ in v. 5 für d'ici setzt, und de toi in v. 6.

Ohne plausiblen Grund fehlen:

$5,9-12 ; 8,7-20 ; 10,9-16 ; 15,7-8 ; 16,17-18 ; 25,3-4$; $28,23-24 ; 31,11-12 ; 41,5-8 ; 44,1-2 ; 44,19-20 ; 55,9-12$; $60,9-10 ; 64,13-14 ; 78,19-22 ; 79,1-80,2 ; 80,7-8 ; 84,21-22$; $87,1-2 ; 92,7-8 ; 92,11-14 ; 101,21-22 ; 108,5-6 ;$ I I $2,7-8$, weshalb i I 2,6 geändert werden mufste zu:

del siecle n'avoit gaires cure;

I I 3,23-24; I I 7,2 I-I I 8,2.

Absichtlich scheinen gekürzt zu sein: 9,23-10,2 zu 2 Versen:

car or les quide avoir sospris, et en ses mains lacies et mis;

75,9-1 $2 \mathrm{zu} 2$ Versen:

dites moi tost, ou il vos tient, quant il vos prist, n'en celes nient;

$105,2 \mathrm{I}-24 \mathrm{zu} 2$ Versen:

molt aves grant merveille dit, onques nus d'aus ainc ne me vit; 
Andererseits zeigt $A_{2}$ viele Hinzufügungen und $Z$ erdehnungen.

I $8,3-4$ zu 4 Versen ezweitert:

cil font sëurté al segnor, s'il ne revient de cele honor, feront a sa soror homage et tenront a grant seignorage;

hinter 24,18 sind 2 Verse eingeschoben: parfondement vait sospirant, et puis si a parlé itant;

2 Verse hinter 26,22:

quant la dame oï la novele, li cuers li bat sor la mamele;

2 Verse hinter 55,1 2:

dont font les lis apareillier, lassé furent, et vont colchier;

2 Verse hinter 80,20:

dont aura il de nos merci, ce sai je bien trestot de fi.

Der Schreiber hatte nicht gemerkt, dafs v. 19 und 20 als nähere Bestimmung zum Vorhergehenden gehörten. Er glaubte, mit v. 19 bgänne ein neuer Satz, dem der Hauptsatz fehlte. Einen solchen schob er deshalb ein.

2 Verse hinter 87,4 :

se li desagree forment

ce que cil li dist laidement;

2 Verse hintert 90,1 2:

dolante fu et tres pensée, et de mal talent molt troblée;

2 Verse hinter 107,10:

lie et joiant tant sojornerent

2 Verse hinter I 13, 17:

que alquens le resvigorerent;

2 Verse hinter I 16,24 :

proies lui que secors vos face, et que il vos otreit sa grace;

tos jors ert mais en memoire, encor l'apele l'on Gregoire.

Endlich sind zu nennen Umstellungen:

7,9-1 2 stehen in der Reihenfolge I 2, I 1, 9, Io mit der deutlich erkennbaren Absicht, durch Schaffung einer Anaphora kräftigere Wirkung zu erzielen. 9,5-6; 15,21-22 unter Beibehaltung der 2 Anfangswörter; 22,5-14 fälschlich hinter 24,14. Die Reihenfolge ist folgende: Hinter 22,4 stehen 22,15-23, 4, und es folgen unter Auslassung der Verse 23,5-6 die Verse 23,7-24,14. Es fehlen 
alsdann $21,23-22,4$, und es folgen $22,5-10,22,13-14,22,11-$ I2. - Andere Umstellungen sind: 4I,19- 20 hinter 4I, 24; $50, \mathrm{I}-2 ; 53,5-6 ; 55,19-20 ; 59,9-10 ; 58,2 \mathrm{I}-22$ hinter 59,2; $60,1 \mathrm{I}-\mathrm{I} 2 ; 60,13-14 ; 60,15-16 ; 71,3-4 ; 86,3-4 ; 109,23-24 ;$ I $17,7-8$ und geändert $\mathrm{zu}$ :

qui furent el ciel coroné

por les grans biens c'orent ovré.

$\mathrm{Zu}$ erwähnen bleibt schliefslich eine von den andern Handschriften abweichende Stelle, die freilich erst späteren Datums sein mufs, da Handschrift $B_{2}$, die sonst sicher auf $A_{2}$ zurückgeht, hier mit $A_{1} A_{3}$ übereinstimmt. Aus dieser Stelle erfahren wir nämlich, dafs sowohl der Herzog als auch Gregor vor Beginn der Schlacht sich in Schimpfreden ergehen, während in den andern Handschriften der Herzog allein es thut. Diese Abweichung rührt her von dem Ausfall der Verse $61,1 \mathrm{I}-15$, wobei wohl cheval in 61,11 und chevalier in 6I, I 5 im Spiele war. Deshalb mulsten Ànderungen eintreten, und zwar wurde 61 , iO umgestaltet $\mathrm{zu}$ :

Gregoir lie crie erraument,

und v. 6r, 16 zu 4 Versen ausgesponnen:

tornes l'escu, vos est mestiers;

avos le di, dans chevaliers!

Et li dus li a respondu :

Mais vos estes li mal venu.

\section{$\S$ 3. Die Handschrift der Nationalbibliothek zu Paris No. $1545\left(A_{3}\right)$.}

Die Handschrift $A_{3}$ ist im Catalogue des Manuscripts de la Bibliothèque Impériale à Paris, Bd.I 247 folgendermalsen beschrieben: „Sur un feuillet de garde en vélin, des comptes de redevances de divers villages situés aux environs de Remiremont, de 1408. Papier. XV. sic̀cle (Anc. 75882, de la Mare 369).“

Unsere Legende steht in dieser Handschrift auf Fol. $121-136$ und folgt auf "Le livre des Perses anciens" commençant par:

„Ayde, roy Jhesu-Christ,

Pere, Filz et Saint Esprit"s

et finissant par:

„Et qui à cellui regnier donne

les biens qu'en cest siecle lui donne.

Explicit Vita Patrum.“

Die Handschrift liefert einen Text; mit dem man sehr willkürlich verfahren ist. Namentlich gilt dies in Bezug auf Verjüngung der überlieferten Wortformen, wodurch sehr häufig sowohl Metrum als Reim arg verletzt ist. Im Einzelnen ist zu bemerken:

I. Schreibfehler haben sich in den Text eingeschlichen: 
de Griffaigne f. d'Aquitaigne I,3; torment f. comand I 7,8; estre f. erre 18,16; doux f. douze 4I,I5; saint Richier f. sire chiers 46,1 I; soudoiers f. soudoies 68,8 ; ouvrage f. orage 35,5 ; ce verrons f. desevrons 8I, $2 \mathrm{I}$; mestier f. maistre 99,1 2; premierement f. parvienent I 16, I 3 ; viennent $\mathrm{f}$. vivent I I 7, 18 .

II. Benachbarte Wörter veranlassen, dafs richtige Wörter durch falsche verdrängt werden:

maulvaistié maulvaise f. volenté maulvaisé 8,6.

III. Unverstandene Ausdrücke geben Anlals zu Änderungen:

el fälschlich $\mathrm{zu}$ et le aufgelöst 20,4 ; toe feie $\mathrm{zu}$ toutes foiz geändert 7 I, I9.

IV. Verse werden verdrängt durch Rekapitulation anderer.

s'elle par toy n'est avancée 5,20 f. mar fu onques la lasse née in Anschlufs an 5,8.

V. Das Metrum wird entstellt:

I. durch Wechsel von Synonymen:

gabez f. enginnez 10,16; le getterent f. l'empeinstrent 26, I 2 ; mot ne soupira f. mot ne sona 44,3.

2. durch Auslassungen einzelner Wörter:

fut hinter molt 13,8; hons hinter gentilz 16,19; en chief hinter de chief 19,7; o hinter ensemble 49,7; bon hinter eust 58,18; dire puis 11,9 ; et hinter riche 38,7 ; ja hinter que 107,17 ; molt tost hinter fait 91,23; jel am Anfange 94,10.

Daneben ist $A_{3}$ reich an Auslassungen ganzer Verse. Aus Unachtsamkeit und mit Reimstörung fehlt I I6, I I.

Verspaare fehlen:

I. wegen gleichen oder ähnlichen Versanfanges:

47,3-4. Wegen car in 47,2 und 47,5 fielen $2-4$ aus. Ein anderer Abschreiber schuf zu 47, 1 einen neuen Vers:

n'en ourras parler jamais;

64, 19-20 wegen tornez I 8 und torné 20;65, 17-20 wegen trestuit dient in 17 und 21 .

II. wegen gleichen oder ähnlichen Versausganges:

I 8,18-19;30,3-4;33,20-34, I wegen mer 33,20 und 34,2; I06, I 2 und 106,15, weil durch Umstellung die Verse I I, I 2, I 5, I 6 zusammengekommen waren, und diese 4 den Reim auf $\varepsilon$ zeigen; $30,6-9$, weil der Blick des Schreiber von enor in 30,5 auf seignor in 30,10 fiel. Um Verständnis hineinzubringen, änderte nun ein Abschreiber v. 10 in Reminiscenz an 19,2 in: comme la fille leur seigneur.

III. wegen gleicher oder ähnlicher Wörter im Innern der Verse: 
I 4,5-6 wegen molt 6 und 4;53,7-8 wegen borjois 7 und 9; 54,15-16 wegen prendre 16 und $18 ; 59,1-2$ wegen ot bon 58,23 und ot bone 59,1 ; $101,7-8$ wegen jor 7 und 9 .

Ohne erkennbaren Grund fehlen:

16,7-10; 31,7-10; 32,19-20; 33,13-14; 37,17-18; $38,1-2 ; 39,9-10 ; 51,1-2 ; 51,13-24 ; 52,13-14 ; 57,17-20 ;$ $58,19-10 ; 59,7-12 ; 60,15-18 ; 61,7-62,10 ; 63,13-18 ; 63$, 13-18; 63,21-22; 64,9-10;69,7-8; 78,9-10; 81,1-2; $84,15-16 ; 90,9-10 ; 91,11-12 ; 92,13-14 ; 93,13-16 ; 98$, 23-99,2; I04,9-10; I09,5-6; II $3,1-2 ;$ I I $4,19-20 ; 116$, $\mathrm{I}-4$.

Absichtlich sind gekürzt: 29,4-6 zu I Vers: hors de ce lict yus la poterent;

29,19-22 zu 2 Versen: quant la nouvelle a entendue, le corps d'angoisse ly tressue;

67,7 - ro zu 2 Versen:

ya soit ce que vous ne m'amez, si vous dis ye que me prenez;

\section{5, I9-2 I zu I Vers:}

s'il laissent aux ondes mener.

Es fehlt aber der hierauf reimende Vers.

Zusätze und Zerdehnungen:

2 Verse hinter 2,6:

et remambrance y veuilliez prendre,

a Dieu pourrez voz ames rendre;

8, I7-I 8 zu 4 Versen zerdehnt, indem für v. I 83 Verse gesetzt werden:

ce est deables, qui sa cure a toute mise a eulx decoivre que de pechié ne fussent sovere;

18, I-2 zu 22 Versen:

adonc ses escripvains manda, lectres fist faire, et seella.

Puis sont venuz les messagiers a cui furent bailliés les briefz.

Par la terre les envoya, et partout ses barons manda qu'a luy viennent hastivement, nel mectent en delaiement.

Quant voient les briefs leur seignour, a luy sont venuz par amour. Quant sont venuz, moustré leur a que en Jherusalem ira, mais avis vient de toute l'onnour 
devant vous saisir ma serour. Bien scay que Dieu a regardé de moy toute ma volenté;

faire de moy puet son plaisir, ou de vivre ou de mourir.

Quel qu'aviengne ne quoy que non, vueil que tout deviengne si hon. Tuit l'octroient yoyeusement, fors tant que de lui sont dolans.

Hinter 23, I5-I8, die in $A_{3}$ lauten: et en chatel doint a l'enfant l'or et le paille reluisant et ses tables qui sont d'ivoire, ou est de ly escript l'istoire,

sind 4 Verse eingeschoben:

pour Dieu requiert ceste dolante qui tant est en doleur $p$ te, que qui les tables trouvera, et l'escript dedens entendra,

und v. I9 und $20 \mathrm{zu} 4$ Versen ausgesponnen: pour Dieu le garde itant de temps que autant ait apris de sens - $\quad$ ly enffes, qui moult est petit.

Dieu gardes le de tous perils;

24,7-8 zu 6 Versen zerdehnt:

le regarda; puis dit itant:

Fils, n'est huy nulle mere vivant tant ait douleur ne esmaiance, comme ye ai par la puissance au diable, qui m'engina tant que mon frere me coucha;

33, 12 zu 3 Versen:

fu es ondes de mer getez, et mis en mer en ung batel, bien enclos dedens ung tonnel;

36,3-6 zu 6 Versen:

il demanda que ce estoit illec dedens, et que devoit que ilz ne trayoient avant; et ly ung dit: ne vous est tant de chose que dedans sachions, car il n'y a, se moult peu non.

69,23-24 zu 4 Versen:

scavoir se ya nul lieu trovast ou il secretement cachast 
les tables, ou estoit escript

qui de luy la verité dit;

78,2 zu 3 Versen:

car mieulx voulsist morir son veul

la comtesse que estre vive.

Plus devint verd que nen est cive;

90, I 7 zu 3 Versen:

quant herbergiez l'avez huy mais,

car le laisses estre en pais

ennuit mais en vostre maison;

2 Verse hinter 95,24:

quant ce ot fait, n'y demora,

entre en la nef, si s'en ala,

tautologisch zu v. 95, 16 , der in in $A_{3}$ lautet:

retorner s'en vint en sa nef;

2 Verse hinter 97,10:

trestous les chrestiens du monde

en sa poëste toujours sont,

tautalogisch zu $97, \mathrm{I}$ I.

Umstellungen:

$28,5-6 ; 31,19-32,16$ in der Reihenfolge: $(32,3-6 ; 32$, I3-16;31,19-32,2;32,7-12); 43,13-14 hinter 43,16; 43, $17-18 ; 75,17-18$ hinter 75,$20 ; 75,23-24 ; 96,19-20$ hinter 96,$24 ; 99,9-12$ hinter $99,13-14 ; 100,5-6 ; 101,9-10$ hinter IOI, I 2 wegen der Auslassung der Verse 101,7-8; 106,9-16 in der Reihenfolge: I3, I 4, 9, I0, I I, I6 (I 2 und I5 fehlen); I I 2, $7-8$.

\section{§ 4. Die Londoner Handschrift $\left(B_{1}\right)$.}

Auf die Handschrift $B_{1}$ hat zuerst Hugo Bieling aufmerksam gemacht in Herrig's Archiv $47,45^{2}$, alsdann sich weiter über dieselbe ausgelassen in seiner Schrift: „Ein Beitrag zur Überlieferung der Gregor-Legende, Berlin 1874." Aulserdem findet man eine Beschreibung bei Neuhaus: „Adgar's Marienlieder, Heilbronn 1886“ (erschienen als Band IX von Förster's Altfranzösischer Bibliothek).

Die Handschrift befindet sich im Britischen Museum zu London und trägt die Bezeichnung Bibl. Eg. 6r2. Im Spezialkataloge ist sie folgendermalsen beschrieben: "Contes dévots consisting of legendary stories, narratives of miracles and lives of saints in early Anglo-Norman poetry; among them are the legends of S. Theofilie and Gregorie. On vellum. Written about 1300 . Imperfect at the beginning and end. Purchased at Sotheby's 1836. Had previously belonged to Wm. Bentham, Esq. of Gower St."

Sie ist eine schöne gut lesbare Pergamenthandschrift und nach Bieling's Annahme entweder Ende des 12. oder Anfang des 
I 3. Jahrhunderts geschrieben. Das Manuskript, in England verfafst, ist in klein $4^{0}$ und enthält auf jeder Seite doppelte Kolumnen. Unsere Legende steht auf Fol. 75c-96a.

Die Fehler dieser Handschrift haben darin ihren Grund:

I. dals sich der Kopist verschrieben hat:

serva f. servi 13,10; ka a f. ke a 16,24; que Rome f. qu'a none 74,22 ; as leis f. esleis 74,9; mot f. maus 28,7; les mains f. le mes 29,7; deserile f. deserte 32,23; de luinz f. de laienz Fol. 87c9; prisun f. prisunier Fol. $87^{\mathrm{c}} 25$.

II. dafs Wörter aus andern Versen wiederholt werden: quident $\mathrm{f}$. puissent $2, \mathrm{I} 6$ wegen quident $2, \mathrm{I} 5$; jo sui f. sui $5 \mathrm{I}, 24$ aus 23 ; ke f. ne am Anfange Fol. $85^{\mathrm{b}} \mathrm{I}_{\mathrm{O}}$ aus $85^{\mathrm{b}} 9$; vie f. ä̈e $9^{\mathrm{d}} \mathrm{I}$ aus Fol. $90{ }^{25}$.

III. Sie bewirken dafs das Metrum gestört wird:

I. wegen Vertauschung von Synonymen:

sèurance f. sëurte 17,2 ; esgarder f. regarder 89,17 ;

2. wegen Gebrauchs eines Simplex für ein Kompositum: trovée f. retrovée Fol. $94^{\mathrm{b}} 5$. umgekehrt:

3. wegen Ersatzes längerer Formen durch kürzere oder

espenir f. espenëir Fol. 92d 6; marchant f. marchaant Fol. 91d I 8; pechur f. pecheur Fol. 9Ic 25; peschur f. pescheur 4I,I9; Fol. 9 IC I5, $^{\mathrm{C}}$

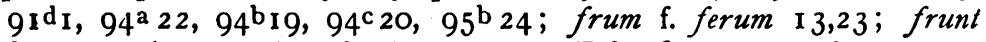
f. ferunt 67,12; frai f. ferai 40,1 I, Fol. 78a 23 ; ore f. or 4,23; 1 3.5; 16,20; 38,11; 46,15;71,19; Fol. 90b 24; Fol. 96a I1 ; encore f. encor Fol. $85^{\mathrm{c}} 22,9 \mathrm{I}^{\mathrm{h}} \mathrm{I} 4,94^{\mathrm{d}} \mathrm{I}$.

4. wegen verjüngter Femininbildung:

queles noveles f. quels noveles 73,19.

5. wegen Auslassung einzelner Wörter:

en hinter ke 5,1; a hinter deit 6,18; de hinter et 10,24; et vor fort 17,9 ; $i$ vor cunduirai 17,11 ; mult vor tost 18,1 ; fud hinter mult Fol. $79^{\mathrm{d}} 3$.

Von einzelnen Versen fehlen:

21,$17 ; 32,2 ; 43,22$.

Von Verspaaren fehlen:

44,3-4 und 75,17-18. Letztere, weil der Schreiber durch diese Verse die ursprüngliche Lesart der Verse 73,9-10 verdrängte.

Umgestellt sind ohne Störung die Verse:

18,1 I-14 hinter 18,8, mit Störung die Verse Fol. 91 ${ }^{\mathrm{a}} 7-8$.

\section{§ 5. Die Arsenalhandschrift No. $325\left(B_{2}\right)$.}

Wie auf $A_{1}$ so machte auch auf $B_{2}$ Luzarche zuerst aufmerksam. Sie befindet sich in der Arsenal-Bibliothek zu Paris und bildet einen Teil der Handschriften-Sammlung Belles-Lettres fran- 
çaises No. 325. Unsere Legende steht dort Fol. $155^{\mathrm{d}}-169^{\mathrm{d}}$ und ist überschrieben:

\section{C'est la vie Saint Gregoire \\ ki fu apostoiles de Rome.}

Die Handschrift ist an einigen Stellen teilweise verletzt, beklebt oder befleckt. So: Fol. 156b 39-43; Fol. 156c 38-44; Fol. 16 Id 18; Fol. $161^{d} 38$; Fol. $165^{d} 56$; Fol. $162 \mathrm{c} 28-34$; Fol. $169^{d} 7$.

Der Dialekt ist der pikardische.

Die Quellen der Verderbnisse liegen:

I. in Verschreibungen:

tel f. tes Fol. $157^{\mathrm{c}} 2$; va f. veit 43,9; enbrasé f. esbrase 8,22; fils f. fille $16 \mathrm{Ib}_{16} 6$; lues f. jus $16 \mathrm{Ib}_{22}$; s'aine f. s'ame $16 \mathrm{Ib}^{\mathrm{b}} 43$; les f. ses $\mathrm{I}_{6 \mathrm{Ic}} 9$; consella f. conrea $\mathrm{I} 6 \mathrm{Id} 40$; si venrons f. desevrons $8 \mathrm{I}, 2 \mathrm{I}$; sainte f. s'ante I I 2,13; cuer talent f. cuer dolent $1_{67} 7_{1}{ }_{5}$; le seneschal et la contesse $55,3 \mathrm{f}$. le seneschal a la contesse, bewiesen durch den Relativsatz:

qui droit a li vos conduira.

II. darin, dafs Wörter aus andern Versen wiederholt werden, oder verursachen, dafs ursprüngliche Wörter eine unrichtige Gestalt bekommen:

femme f. frere 45,11 aus 45,10 ; dire f. lire Fol. $165^{\text {a } 10}$ aus I65a9; diex f. ciex I09, 8 aus I09, I7; sera mort f. se racort II I; I4 in Anschluls an sera mort 111,13 ; assez f. altre 27,6 aus 27,5; parfui f. effui I 5,20 wegen paroï I 5, 19; reconforter f. por conforter 21,7 wegen reconte 21,6; par penitance Fol. $165^{\mathrm{c}} 47$ f. a penitance wegen par sa pitance Fol. $165^{\mathrm{c}} 4^{6}$; coment f. ainz ke Fol. $165^{\mathrm{C}} 59$ wegen coment Fol. $165^{\mathrm{c}} 57$.

III. darin, dafs Wörter nicht verstanden wurden:

Daher maus fait fälschlich zu mesfait geändert 28,7 .

IV. darin, dals das Metrum gestört wird durch Auslassung einzelner Wörter:

fils hinter biaus 4,19; ne vor eust 59,16; li hinter il 63,6; ne vor tire 73,15 ; il hinter dist Fol. $16 \mathrm{I}^{2} 32 ; a$ vor ambedeus Fol. $159^{c} 14$.

$\mathrm{Zu}$ diesen Auslassungen einzelner Wörter treten Auslassungen ganzer Verse. Von einzelnen Versen fehlen:

7,$8 ; 58,17$; der Vers $B_{1} 8 g^{c}{ }_{1} 2$ und 1 Vers hinter Fol. $167^{b}{ }_{27}$.

Fehlen Verspaare, so kann diesen Ausfall bewirkt aben:

I. gleicher Versanfang:

$B_{1} 82 \mathrm{~d} 8-9$ wegen $e t$ in 8 und 10 ; deshalb v. 10 und II $\left(B_{2}\right.$ Fol. 160a 25-26) geändert, so dafs sie in die Konstruktion passen. $\mathrm{B}_{1}$ liest:

e si acunta a sa gent

ke de sa fille est veirement,

Zeitschr. f. roin Phil. $\mathbf{X}$. 


\section{Dafür $\mathrm{B}_{2}$ :}

si l'enveia a dan abé

ke li donast chrestienté.

et puis l'envoient bautisier

a dant abé en son moustier.

Dann fehlen die Verse $B_{1} 84^{d} 24-25$ wegen et $84^{d} 24$ und $85^{\text {a I }}$. Endlich sind ursprünglich ausgefallen die Verse 62,9-I 2 wegen quant $\mathrm{B}_{1} 89^{\mathrm{a}} 9$ und $\mathrm{I} 3$. Da hierdurch eine Lücke entstand, schob $B_{2}$ (Fol. 164d 8-9) 2 Verse ein und ändert in $164^{d} 10$ das quant la von $\mathrm{B}_{1}$ zu se li.

II. gleicher oder ähnlicher Versausgang:

So die Verse $B_{1}\left(9_{2} d_{16}-21\right.$ (in $B_{2}$ zwischen $166 d 44$ und 45) wegen veir (verum) $92 \mathrm{~d}_{1} 5$ und veer (videre) $2 \mathrm{I}$.

III. gleiche Wörter im Innern der Verse:

So die Verse $B_{1} 9^{c} 7-8$. Die Verse $92^{c} 6$ und ro enthalten nämlich das Wiort eve. Aus diesem Grunde fielen 7-10 aus. Da hierdurch eine Lücke entstand, fügte später ein Abschreiber von $B_{2} 2$ Verse selbständig ein $\left(B_{2} I_{66} d_{1} I-12\right)$.

Ohne plausiblen Grund fehlen:

$\mathrm{B}_{1} 79^{\mathrm{a}} 8-9 ; \mathrm{B}_{1} 82^{\mathrm{a}} 23-24 ; \mathrm{B}_{1} \mathrm{O}_{\mathrm{d}} 4-\mathrm{I}_{5} ; \mathrm{B}_{1} 93^{\mathrm{d}} \mathrm{I}_{8}-19$; p. 109,7-8; die in $A_{2} A_{3}$ enthaltenen Verse 109, I $4 a$ und $b$, welche dort lauten:

et quant il entra dedens Rome, tous li clergies et li autre home;

dann 8 Verse zwischen $1 \mathrm{II}, 4$ und 5 , die $\mathrm{A}_{2} \mathrm{~A}_{3}$ aufweisen:

$$
\begin{aligned}
& \text { quant il ot receu l'onor } \\
& \text { si con il plot al creator, } \\
& \text { de dieu proier ne se targea, } \\
& \text { mais plus en plus s'en efforça, } \\
& \text { et diex qui les siens pas n'oblie, } \\
& \text { son bon sergeant n'oblia mie; } \\
& \text { abandon li fist de son bien, } \\
& \text { por lui salva maint crestien. }
\end{aligned}
$$

Endlich die Verse I 14,19-22; 115,9-10; 1 16,17-18; 116, $21-117,18$.

Zahlreich wie diese Lücken sind nun anderseits $\mathrm{Zu}$ sätze:

2 Verse hinter 3,2:

Hui mais ores le grant dolor

que puis avint au pecheor;

2 Verse hinter 45,16 die tautologisch $\mathrm{zu} 45,17-18$ sind:

li abes fu molt sages hom,

si quist celui en sa maison;

2 Verse hinter 62,22: 
parmi l'escut paint a lion

li fait passer le gonfanon;

2 Verse hinter $B_{1} 87^{\mathrm{b}} 25$, die stören und Rekapitulation sind aus $64,9-10$ :

a vive force $0 \mathrm{mal}$ talent

molt les menoient laidemeut;

2 Verse hinter $B_{1} 87 d_{2}$ :

ensi s'en fait tost reporter

en son païs, por respasser;

2 Verse hinter 72,1 2:

car jou me vuel caens estier

tot solement, por deporter;

2 Verse hinter 72,24:

se damediex ne le tenist,

ja en son sens ne remansist;

2 Verse hinter 73,20:

avois hucé li escuiers,

ahi quel duel, frans chevaliers!

2 Verse hinter 74,5 :

mais Gregoires qui la dame aime,

et qui de li servir se paine

unter Änderung des v. $6 \mathrm{zu}$ :

torne le cief de son ceval;

2 Verse hinter $B_{1} 90^{2} 10$ :

de cest forfait orons la vie

dont l'estoire est chaens escrite;

2 Verse hinter $B_{1}$ god 18 :

quant iceste corte leeche

nos revenra a grant tristeche;

hinter $B_{1} 93^{c} 5-6$, die in $B_{1}$ lauten:

unkes nuls hoem nel regarda,

neis li culvert qui l'ensera

2 Verse:

n'en souvint onques nule fois.

Or sachies bien que fu destrois.

Der Schreiber merkte nicht, dafs v. $\mathrm{B}_{1} 93^{\mathrm{c}} 6$ auch von regarda abhing. Er glaubte, mit neis begänne ein neuer Satz, dem das Verbum fehlte.

2 Verse hinter 108,24:

devant la porte humlement

depria Dieu omnipotent,

die tautologisch zu 108,24 und 109,2 stehen, denn 109, $1-2$ liest $\mathrm{B}_{2}$ :

envers le ciel un regart fist,

depria Dieu, et se li dist;

2 Verse hinter 109,2: 
glorieus diex qui me fesis,

et en mon cors ame mesis;

die Verse 109,19-20; 2 Verse hinter I I 3,22:

les pies li baise, et si l'acole,

or li plaist molt icele escole,

(erster Vers tautologisch zu I I 3,23:

estroitement les pies li baise);

2 Verse hinter II 5,20:

por ses pechies espeneïr,

juner, villier et peu dormir;

endlich die Verse I I 8,7-I 2 .

An diese Zusätze schliefsen sich eine grofse Anzahl Zerdchnungen.

43,10-1 2 zu 5 Versen:

se li commence a demander

qui içou li avoit mesfait.

Il dist: Gregoires li a fait,

et cele molt tost si s'ecrie

a haute voiz con esmarie;

59,3-4 zu 6 Versen:

cauces de fer molt biens ouvrées,

et en ses jambes bien fremées.

$\mathrm{Li}$ esporon furent a or,

en Egypte les fisent Mor.

Li bons abes se li dona,

quant a chevalier l'adouba;

die Verse $B_{1} 89^{c} 23-89^{d} 3$ zu 8 Versen. $B_{1}$ liest:

sire, dist ele, dulce vie :

e dites ore a vostre amie

pur amur deu, le rei celestre,

ainz que jo muere, de vostre estre,

de quel pais fud vostre mere,

e quel hoem fud vostre pere.

Die Abweichung in $B_{2}$ ist so zu erklären: Für amie hatte ein Schreiber vie aus dem vorhergehenden Verse genommen. Dadurch wurde die Konstruktion gestört. Ein zweiter Abschreiber änderte ainz que jo muere $\mathrm{zu}$ car diles ore und verarbeitete den in den Wörter ainz que jo muere enthaltenen Gedanken in 2 Versen, die er hinter $B_{1} 89^{d} 3$ einschob:

me dites ore ainz que jo muire

vos le metes molt taisant cuire.

$B_{1} 93^{d}$ I 3 zu 3 Versen:

ens el sepulcre le poserent

molt gentement con lor signor,

con lor empereor pastor;

die Verse $B_{1} 94^{2} 9-10$ : 
li clerc alerent tant querant

ke un matin vindrent errant

zu 6 Versen:

$$
\begin{aligned}
& \text { par cel saintisme anoncement } \\
& \text { que diex noncha en lor convent, } \\
& \text { montent dui clerc de la cité; } \\
& \text { en lor cemin en sont entré. } \\
& \text { Un jor et autre quisent tant } \\
& \text { c'un matinet a l'ajornant. }
\end{aligned}
$$

$\mathrm{Da}$ aber die Handschrift den nächsten Vers wie $\mathrm{B}_{\mathfrak{l}}$ liest, nämlich

en la maisun d'un pescheur,

fehlt das Verbum zu $167^{c} 29$.

Die Verse $B_{1} 94^{a}$ I $8-20$ :

un grant (i. e. poisson) lur ad devant porté,

e cil l'unt mult tost acheté,

si l'en donent plus que le dreit

zu 5 Versen:

cil en quisent a acater.

Il lor en fait un aporter, qui molt par fu et bons et chiers, et cil l'en donent volentiers asses avant outre le droit;

die Verse $B_{1} 94^{\text {a } 22-23: ~}$

si unt prié le pescheur

k'il lur cunreit pur deu amur

zu 4 Versen:

quant eslegié ont le poisson, pus si deprient au baron qu'il lor conroit par veritet trestot le miex qu'il onques set;

die Verse $B_{1} 94^{d}$ /4-i 5 :

cil surent bien demaintenant que ceo ert cil qu'il vunt querant

zu 6 Versen:

si tost come li clerc le virent trestot ensamble tres bien dirent que cou ert cil qu'il vont querant, et si en orent oï tant le pescheor dire et conter qu'il n'en vaurrent plus demander;

I 1 3,24 zu 3 Versen:

por cou ke lieu en a et aaise;

onques li cors ne li apaise, ce li semble que trop se taise;!

I 14, I 4 zu 3 Versen: 
quant damediex, qui m'a crié,

a ma joie m'a ramenée

que jou avoie entroublée.

Umgestellt sind:

42,19-20; I1 4,6-7; $\mathrm{B}_{1}$ Fol. $83^{\mathrm{d}} 22-23$ und $\mathrm{B}_{1} 87^{\mathrm{a}} 22-23$.

\section{TEIL II.}

GRUPPIERUNG DER HANDSCHRIFTEN.

$\S$ I. Keine der Handschriften ist Original, oder war Vorlage der anderen.

$A_{1}$ hat allein die Lücken hinter 42, I I und 72,14; $A_{2}$ allein die Lücken $20,23-24 ; 45,7-14 ; 53,11-12 ; A_{3}$ allein die Lücken $37,17-18 ; 39,9-10 ; B_{1}$ allein die Lücke 44,3-4; $B_{2}$ den AHandschriften gegenüber allein die Lücke $62,9-12, B_{1}$ gegenüber die Schilderung der Sühne der Mutter.

\section{$\S$ 2. Die Handschriften teilen sich in eine A- und B-Gruppe.}

Inhaltlich sowohl, wie in formeller Beziehung zerfallen die uns vorliegenden Handschriften in 2 scharf abgegrenzte Gruppen A und B. $\mathrm{Zu}$ der ersten gehören die mit $\mathrm{A}_{1} \mathrm{~A}_{2} \mathrm{~A}_{3}$, zur zweiten die mit $\mathrm{B}_{1} \mathrm{~B}_{2}$ bezeichneten Handschriften. Berücksichtige ich an dieser Stelle nur die formale Seite, so unterscheidet sich die B-Gruppe von der A-Gruppe.

I. durch Kürzungen, die durchweg anzutreffen sind:

$$
\begin{aligned}
& \text { 3,1 7-20 entsprechen }\left\{\begin{array}{lll}
B_{1} & 76 a_{2}-3 ; \\
B_{2} & 156 a_{43}-44 ;
\end{array}\right. \\
& \text { 5,23-6,4 entspr. } \quad\left\{B_{1} 76 \mathrm{~b}\right. \text { 19-10; }
\end{aligned}
$$

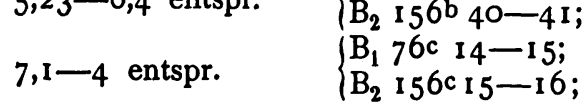

$$
\begin{aligned}
& \text { 8,1 7-20 entspr. } \quad\left\{\begin{array}{l}
B_{1} 77^{a} 2-3 ; \\
B_{2} 156 d 8-9 ;
\end{array}\right. \\
& \text { 9.2 I-24 entspr. } \quad\left\{\begin{array}{l}
B_{1} 77^{a} 24-25 ; \\
B_{2} 156 d 30-31 ;
\end{array}\right. \\
& \text { I 1,9-1 2, I } 2 \text { entspr. } \quad\left\{\begin{array}{l}
B_{1} 77^{c} 6-11 ; \\
B_{2} 157^{a} 17-22 ;
\end{array}\right. \\
& \text { I 4,3-10 entspr. } \quad\left\{B_{1} 78 \text { a } 2-3\right. \text {; } \\
& \left\{\begin{array}{l}
\mathrm{B}_{2} \mathrm{I} 57^{\mathrm{b}} 20-2 \mathrm{I} \\
\mathrm{B}_{1} 78 \mathrm{a} \text { I } 8-23
\end{array}\right. \\
& \text { 15,3-14 entspr. } \quad\left\{\begin{array}{l}
B_{1} 78 a_{1} 8-23 ; \\
B_{2} 157^{b} 42-157^{c} 4 ;
\end{array}\right. \\
& \text { 25,23-16,18 entspr. }\left\{\begin{array}{l}
B_{1} 78 b 9-22 ; \\
B_{2} 1_{57} c^{c_{3}-26} .
\end{array}\right.
\end{aligned}
$$

u. s. w. 
II. durch $Z$ usätze und Erweiterungen:

$$
\begin{aligned}
& \text { 1 2, 17-22 entspr. } \quad\left\{\begin{array}{l}
B_{1} 77^{c} 16-23 \\
B_{2} 157^{a} 27-34 ;
\end{array}\right. \\
& \text { 24,5-10 entspr. } \quad\left\{\begin{array}{l}
\mathrm{B}_{1} 79^{\mathrm{d}} 25-8 \mathrm{O}^{\mathrm{a}} 7 \\
\mathrm{~B}_{2} 5^{8 \mathrm{c}} 3 \text { - } 10 ;
\end{array}\right. \\
& 46,20 \text { entspr. } \quad\left\{\begin{array}{l}
B_{1} 84^{a} 8-10 \\
B_{2} \text { I } 6 \text { od } 28-30 ;
\end{array}\right. \\
& \text { 48, 1 } 9-20 \text { entspr. } \quad\left\{\begin{array}{l}
B_{1} 84^{b} 20-25 \\
B_{2} \text { I } 6 \text { I } 2 \text { I I-26; }
\end{array}\right. \\
& \text { 50,15-5 I,2 entspr. } \quad\left\{\begin{array}{l}
\mathrm{B}_{1} 84^{\mathrm{d}} \mathrm{I} 2-85^{\mathrm{b}} \mathrm{I} \\
\mathrm{B}_{2} \mathrm{I} 6 \mathrm{I}^{\mathrm{b}} 22-\mathrm{I} 6 \mathrm{Ic} ;
\end{array}\right.
\end{aligned}
$$

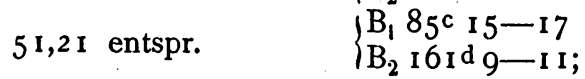

$$
\begin{aligned}
& \text { 54,23 entspr. } \quad\left\{\begin{array}{l}
B_{1} 86 b 6-8 \\
B_{2} \text { I } 62 a 29-31 ;
\end{array}\right. \\
& \text { 59,9-1 } 2 \text { entspr. } \quad\left\{\begin{array}{l}
B_{1} 86 b_{20}-25 \\
B_{2} I_{2} 2^{a} 43-162^{b} b_{3} .
\end{array}\right.
\end{aligned}
$$

u. s. w.

III. durch gemeinsame Umstellungen: hinter 86,18 .

$7,17-18 ; 23,15-16 ; 38,15-16$ hinter 38,$20 ; 86,15-16$

\section{§ 3. $B_{2}$ eine Mischhandschrift.}

Obgleich nun auch zum grölsten Teil $B_{2}$ in ihren Lesarten mit $B_{1}$ übereinstimmt, so zeigt sie doch an mehreren Stellen die Eigentümlichkeit, dafs sie B- und A-Lesarten vermischt, dals also der Schreiber neben einer B-Vorlage eine A-Vorlage benutzte. Am deutlichsten sieht man dies in den einzelnen Teilen der Kampfscene, weshalb ich diese Scene auch zuerst betrachten will.

In den Versen $57,21-58,16$ wird uns die Eröffnung des Kampfes geschildert. $B_{1}\left(\right.$ fol. $\left.87^{a}{ }_{1}-6\right)$ kürzt diese Scene zu 6 Versen :

$$
\begin{aligned}
& \text { anceis que miedi fust passé } \\
& \text { es vus l'assalt a la cité. } \\
& \text { Un riche ducs l'ad assegée, } \\
& \text { ki lungement l'ad guerrée, } \\
& \text { Icels dedenz s'adubent tost } \\
& \text { ki turneer s'en vont en l'ost. }
\end{aligned}
$$

$B_{2}$ (fol. $162 \mathrm{c} 9-28$ ) hingegen hat 20 mit den A-Handschriften gleich lautende Verse.

Die v. 58, 17 und 18 sind $A$ und $B$ gemeinsam. Die folgenden

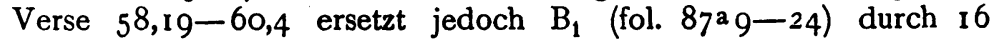
selbständige Verse:

Gregorie fud mult enginnus

e des membres bien vertuus. 
Devant les altres esporone, mult roistes cops $i$ fiert e dune.

De la lance que li ber porte la hanste fud e dreite $e$ forte k'il n'a consiut cel chevalier ke li n'estuce trebuchier. Gregorie fud sages li ber, e si fud mult bon chevalier alkes par sa grant sapience e alkes par sa grant puissance, se set si bien de tut garder ke nuls nel poet de rien grever ne pur ferir ne pur buter.

Tant se saveit bien demener.

$\mathrm{B}_{2}$ indessen lärst die 4 ersten Verse von $B_{1}$ unberücksichtigt und giebt dafür (fol. $1_{62}{ }_{30}-162^{d}{ }_{23}$ ) alle die Verse, welche $A$ aufweist. Dann kehrt sie zu ihrer B-Vorlage zurück und nimmt (fol. I62d 24-35) die I 2 Verse auf, die $B_{1}$ (fol. $87^{\text {a }}$ 13-24) zeigt. Dadurch werden die Verse 58,23 und 59,22-23 zwei Mal gebracht, einmal in der Gestalt, wie sie die A-Handschriften aufweisen, das zweite Mal, wie sie $B_{1}$ liest. So liest $B_{2}$ ( I $\left.62 c 34\right)^{2}$ gemäls A :

hauberc ot bon et lance forte

und (fol. 162d 24-25) gemäls B :

et li lance que li bers porte,

fu si tres fors et rade et forte,

(die nur eine Verschlechterung der Lesart $B_{1}\left(87^{a} I_{3}-14\right)$ sind), anderseits die v. 59,22-23 gemäls $A\left(B_{2}\right.$ fol. $\left.162^{d}{ }_{17}-18\right)$ :

pluisor en a fait trebuchier;

cil cui consieut, a fait verser,

und $\left(162^{\mathrm{d}} 25-26\right)$ gemäls $B$ :

que il ne consieut chevalier

que il ne face trebuchier.

In den Versen 6o,5-25 gehen nun $B_{1}$ (fol. $87^{a} 25-87^{b} 9$ ) und $B_{2}$. (fol. $162 d{ }^{2} 6-43$ ) zusammen. Gemeinschaftlich ändern sie hierauf die Verse $6 \mathrm{r}, \mathrm{I}-2 \mathrm{zu}$ :

quant Gregoire le mot entent, l'escu embrace fierement,

und fügen hierhinter 4 Verse. Darauf weicht von $6 \mathrm{I}, 3-65,6 \mathrm{~B}_{1}$ (fol. $87 \mathrm{~b}$ r6-23) vollständig von $\mathrm{A}$ ab und liest 8 Verse:

Gregorie ad le duc feru

el descovert defors l'escu

si que par mi l'alberc blanc

li mist le fer al destre flanc.

Quant vers terre le veit verser, si l'embrace par mi li ber. 
Puis l'ad saché laenz si tost

ke unkes n'ot socurs del host.

$B_{2}$ hingegen setzt in die Lesart von $A$ mit v. 6r,2 ein und geht genau von Fol. $163^{a} 6-165^{c} 20$ mit diesen Handschriften. Vor 6 I,2 schiebt sie nur I Vers ein:

Gregoires a le duc vëu,

der entweder für $6 \mathrm{I}, \mathrm{I}$ steht und geändert ist, weil dieser Vers in B schon vorher in der Gestalt:

quant Gregorie le mot entent

vorkommt, oder $A_{2}$ aus v. 60,19 entlehnt ist. Dieses Verses wegen werden die in $B_{1}\left(87^{b} 4-9\right)$ stehenden Verse:

Gregorie vit le duc mult fier,

si commença a demander,

ki pot ce estre as armes chieres

ki la conduist ces granz eschieles.

$\mathrm{Li}$ cumpaignun respunent tost:

Ço est li ducs ki conduist l'ost,

von $\mathrm{B}_{2}\left(\mathrm{r}_{2} \mathrm{~d} 4 \mathrm{O}-43\right)$ zu 4 Versen gekürzt und geändert, weil der Schreiber eine Wiederholung vermeiden wollte:

Gregoires a le duc demandé,

et on li a bien avisé

que çou est cil as armes chieres

qui la conduisoit ces banieres.

Nachdem $B_{2}$ den A-Handschriften bis v. 65,6 gefolgt ist, wendet sie sich wieder zu ihrer B-Vorlage. $B_{1}$ hat bis hierhin ganz kurz den Kampf Gregors mit dem Herzoge erzählt, welcher damit endet, dafs der Herzog gefangen genommen und in die Stadt geschleppt wird. Von $87^{\mathrm{b}} 24-87^{\mathrm{c}} 6$ werden nun die Folgen dieser Gefangennahme berichtet. Mit Gregor sind die Bürger in die Stadt gezogen und haben die Thore geschlossen. Die Feinde wollen durch einen Sturm die Stadt nehmen, werden aber zurückgeschlagen. Diese letzte Schilderung nimmt auch $B_{2}$ auf, die vorher mit den A-Handschriften ausführlich den Kampf Gregor's mit dem Herzoge vor der Stadt, sowie einen an des letzteren Gefangennahme sich anschliefsenden Strafsenkampf geschildert hat. $\mathrm{Da}$ sie aber auf diese mit den A-Handschriften gemeinschaftliche Schilderung ohne ein Bindeglied nicht gleich die Verse von $B_{1}$ (f. $87^{b} 24-87^{c}$ c ) folgen lassen kann, schiebt sie hinter die Lesart von A $(65,6)$ erst 6 Verse ein (fol. $163^{c}$ 20-25):

Quant Gregoires les ot mis fors

de la cité par ses esfors,

et il les ot asses cachies

et abatus et detrenchies,

en la cité retorne ariere

ensamble sa compagne fiere,

und geht dann (fol. $163^{c} 26-35$ ) gemeinsam mit $B_{1}$ : 
apres Gregoire sont entré tout li borgois de la cité [a vive force, o mal talent (nur $\mathrm{B}_{2}=64,9-10$ )], molt les menoient laidement

et li sergeant et li meisnie

si ont la porte verouillie;

et li grans bruis de toute l'ost;

les vont suivant apres molt tost;

s'escuident prendre par asaut, mais noient ne monte ne vaut.

Haben wir also in $B_{1}$ neben der Schilderung des Kampfes zwischen Gregor und dem Herzoge vor der Stadt nur die eines Angriffsversuches auf die Stadt, in A nur die eines Strafsenkampfes so haben wir in $\mathrm{B}_{2}$ sowohl den Strafsenkampf als den Angriffsversuch der Feinde auf die Stadt.

In den Versen $65,7-8$ gehen nun $A$ und $B$ zusammen; von 65,9-66,22 weichen jedoch $B_{1}$ (fol. 87c 8-24) und $B_{2}$ (fol. I $63^{c}$ $\left.38-163^{d} 9\right)$ in ihren Lesarten gänzlich von $A$ ab:

Cex de laiens treve ont mandée;

lors s'en revont en lor contreé.

Le duc laissierent en prison, bien ait Gregoire li preudon, qui sa mere a si acuitée, et la guerre si tost finée. Qui dont veïst les citouains a Gregoire baisier les mains et celes dames haut monter por le baron a esgarder, qui par son cors tant seulement a pris un duc devant sa gent! Trestot hucent, grand et petit: Cestui prendes, dame, a marit! $\mathrm{Li}$ dyables les fait parler, qui Gregoire velt tormenter.

Es wird also geschildert, wie die Feinde um Frieden bitten, abziehen und den Herzog in Gefangenschaft lassen, wie dann die befreiten Bürger, erfreut über Gregor's Erfolge, ihrer Herrin zujubeln, sie möge ihn zum Gemahl nehmen. - Nach dieser Schilderung wendet sich $B_{2}$ wieder zu ihrer A-Vorlage, setzt in v. 65, 13 ein und schreibt (fol. 16 $3^{\mathrm{d}}$ 10-41) sämtliche Verse bis 66,22. Dadurch wird die Schilderung des Friedensschliefsens, des Abzuges der Feinde und des Verlangens der Bürger, Gregor als den Gemahl ihrer Gebieterin zu sehen, zwei Mal uns vorgeführt.

Endlich ersetzt $B_{1}$ die Verse 66,23-67,4 durch 2 Verse $\left(87^{c} 25-87^{d}\right.$ i): 
Le prisunier tost desarmerent,

a la dame le presenterent.

$\mathrm{B}_{2}$ (fol. $163^{\mathrm{d}} 40-45$ ) jedoch giebt 6 Verse, wie die A-Handschriften.

Einen neuen Beweis liefern die Verse 42,13-43,8, in denen der Anlals geschildert wird, aus welchem die Frau des armen Fischers dem zwölfährigen Gregor vorwirft, dals er als Findling in das Land gekommen. $B_{1}\left(83^{b} 18-25\right)$ kürzt diese Erzählung zu 8 Versen unter Benutzung der Verse 42, 13-16 und 43,5-8, indem sie v. 42,16 und die Verse 43,5-8 selbständig ändert.

Sie liest:

Il avint chose en cel temporie

ke de duze anz fud Gregorie

un matinet alat juer

sur le rivage de la mer,

e si feri le fiz celui

ke son luier aveit de lui;

si avint que li emfant

vint a maisun tut plurant.

In den ersten 4 Versen schliefst sich $B_{2}\left(160^{\circ} 39-42\right)$ an $B_{1}$, fügt hierhinter $(160 \mathrm{~b} 43-46)$ die in $A(42,17-20)$ enthaltenen 4 Verse, zeigl dann inhaltlich wieder dieselbe Lesart wie $B_{1}\left(83^{b}\right.$ 22-25) und ändert nur die Form, wie es durch den Einschub bedingt wird. So entsprechen den eben genannten 4 Versen von $B_{1}$ in $B_{2}$ die Verse $160^{b} 47-50$, welche lauten:

Par jeu Gregoires feru l'a,

et cil forment s'en coreça,

droit a sa mere vint corant,

se li a dit le convenant.

Inhaltlich weicht somit $B_{2}$ ebenso wie $B_{1}$ von $A$ ab, als beide Handschriften die Art des Spiels unbezeichnet lassen, durch das die Entzweiung zwischen Gregor und dem Fischerssohne zu Stande kommt.

Eine weitere Mischung scheint in $B_{2}$ vorzuliegen in den Versen 53,3-4. $B_{1}$ (fol. $85^{d}{ }_{14}$ ) und $B_{2}$ (fol. 16 Id 33 ) ändern v. 53,3 selbständig in:

Gregorie ist fors de la barche.

Auf diesen lälst dann $B_{\mathfrak{\jmath}}$ an Stelle von 53,4 drei ganz anders lautende Verse folgen:

si vit le pais grant e large

dunt cuntesse esteit sa mere,

ki l'ot ëu de son chier frere,

$\mathrm{B}_{2}$ hingegen liest einen den A-Handschriften gleichen Vers: ot bon destrier et forte targe.

Doch will ich hierauf kein Gewicht legen. Es wäre ja möglich, dals in der Vorlage von $B_{\mathfrak{f}}$ v. 53,5 gefehlt, und dals die da- 
durch entstandene Lücke ein Abschreiber durch 3 Verse ausgefüllt hätte.

Deutlicher erscheint $B_{2}$ wieder als Mischhandschrift in den Versen 87,9-10. Gregor bittet den Fischer, welcher ihn später an den Felsen schmiedete, um Obdach während der Nacht. Dieser will es anfangs nicht gestatten, erklärt sich aber schliefslich auf Ersuchen seiner Frau dazu bereit.

Die Stelle lautet in A $(87,9-10)$ :

quant por Deu te fai le reclaim,

fai le gesir sor cel estraim.

$B_{1}\left(9^{a}\right.$ I $\left.5-16\right)$ ändert diese Verse $z u:$

quant pur deu ostel demanda,

en cel teitun culchum le la.

$\mathrm{B}_{2}$ (fol. I66c 1 2-15) verbindet die Lesart von $\mathrm{A}$ mit der von $B_{1}$. Sie lärst die ersten 2 Verse, wie sie $A$ hat, fügt dahinter die Verse, welche $B_{1}$ aufweist, aber, wie es durch den Vorschub der A-Verse nötig geworden, in veränderter Form. Sie liest deshalb für die Verse von $B_{1}$ :

en cel toitel la dehors soit

con fors pechieres que il soit.

Die Verse 84,19-22 kürzt $B_{1}$ zu 2 Versen:

e si cum povres e chaitis

s'en fui hors del païs,

$B_{2}$ 166a $42-45$ aber hat 4 Verse wie A:

si c'a la loi de mendiant

s'en $\epsilon$ st fui a coc chantant

fors de la chambre le sien pere

e de l'honor qui fu sa mere.

Eine weitere Mischung erscheint in den Versen 83,15-84, 10, wo $\left.B_{1} 9 \mathrm{I}^{\mathrm{b}} 4-25\right)$ und $\mathrm{B}_{2}\left(\mathrm{I}_{4} 6 \mathrm{a}_{20}-39\right)$ bedeutend von $A$ abweichen. In den ersten 8 Versen geht $B_{2}$ mit $A$, fügt dahinter 4 eigene Verse, lärst dann 2 Verse folgen, die $B_{1}$ zu Anfang dieser Schilderung hat, kehrt wieder zu A zurück, liest die Verse 84,9-10, die in $B_{1}$ nicht vorhanden sind und fügt dahinter 2 eigene Verse. So erklärt sich auch die abweichende Lesart von $\mathrm{B}_{2}$ ( $\mathrm{I}_{4}{ }^{\mathrm{b}}$ 28-33) von der in $B_{1}(88 \mathrm{~b} 20-25)$. Gregor sucht einen Ort, wo er seine Tafeln verbergen kann, vermag aber keinen zu finden:

desque ceo vint apres super

ke sul s'en entrat a celée

en une chambre mult privée.

Tant $i$ entrat celeement

ke nul nel saveit de sa gent.

Il regardat un poi ariere etc.

Von dieser $B_{1}$-Lesart behält $B_{2}$ nur den ersten und letzten Vers, die andern ändert sie in Anschlufs an $\mathrm{A}(70,5-8) \mathrm{zu}$ : 
que le cambrelenc apiela;

a le privée cambre ala.

Quant il ot fait la muserie

quant maint hom fait nesarie (?).

Diese Änderung hatte zur Folge, dafs die Verse $B_{1}$ (88c $\left.5-8\right)$ :

e veirement accustumé ad

k'un sul jor ne trespasserad

qu'il ne venist regarder

celeement e tut sans per,

von $B_{2}\left(r_{6} 4^{b} 38-43\right)$ umgestaltet wurden $\mathrm{zu}$ :

si que cil mie ne le vit

qui a la cambre l'ot servit.

Dont s'atorne tout entresait;

onques nul jor ne l'entrelait

qu'il ne reviegne a l'arçon

la ou les tables mises sont.

Durch die Verse in A $(82,5-8)$ sind dann auch wohl die Verse $B_{2}\left(165^{d} 29-3^{2}\right)$ veranlafst worden, die in $B_{1}$ ohne jede Störung hinter god 25 fehlen:

la balance est forment cargie ou si grant coupe iert coucie, ne quid que soit contrepesée

del grignor mont de la contrée,

und so stammen auch wohl die in $B_{1}$ ohne Störung fehlenden Verse $14,13-14 ; 69,1-2 ; 72,24$ und 73,$2 ; 89,21-22$ aus A.

Auf den Umstand, dafs $\mathrm{B}_{2}$ Misch-Handschrift ist, möchte ich auch den Ausfall der Verse 15,21-22 zurückführen, die in $B_{2}$ hinter 15,2 zusammen mit 2 andern Versen stehen und lauten:

si en sospire molt forment,

et si en pleure tenrement.

Li gentius hom ot grant paor, quant a ses pies voit son signor.

Die Verse $15,1-2$ lauten nämlich in $B_{1} B_{2}$ :

nes li frans hom ki ne set mie

que cele chose senefie.

Von diesen Versen wandte sich der Schreiber von $\mathrm{B}_{2} \mathrm{zu}$ seiner A-Vorlage, und wegen der Wort francs hom in 15, I fiel sein Blick auf francs hom in 15,19, weshalb er hinter 15,2 die Verse $15,21-$ 22 folgen liefs. $B_{1}$ und somit wohl auch die Vorlage von $B_{2}$ hat zwar auch die Verse 15,19-20. Hier kann aber das francs hom in 15,19 nicht bewirkt haben, dafs von dem Schreiber von $B_{2}$ die Verse $15,21-22$ hinter 15,2 gestellt wurden, denn $B_{1} B_{2}$ haben hinter 15,20 zwei Verse eingeschoben, mithin wären doch diese hinter 15,2 gesetzt worden. Nachdem der Schreiber von $B_{2}$ diese Verse $A_{2}$ entlehnt und dann eigenmächtig noch 2 andere Verse eingeschoben hatte, kehrte er wieder zu seiner B-Vorlage zurück 
und setzte in v. 15,3 ein. Die Verse $15,21-22$ unterdrückte er alsdann absichtlich, da er sie vorher zu dem Einschube benutzt hatte.

Endlich zeigt sich $\mathrm{B}_{2}$ noch deutlich als Mischhandschrift in der Schilderung der Reise Gregor's nach Rom. $B_{1}$ (95' 23-24) und $B_{2}(168 c 20-2 \mathrm{r})$ sind zusammengegangen bis zu den Versen:

es vus les clerc joyans e liez,

ignelement saillent en piez.

Da bricht $B_{2} a b$, setzt in die $A_{1}$ hinter I08, 6 fehlenden Verse: or n'i vaurront plus demorer icil qui l'en doivent mener

ein und zeigt die ausführliche Reiseschilderung, wie sie in A bis v. I I I,20 steht. $B_{1}$ jedoch kürzt die Reiseschilderung zu 2 Versen:

tut dreit a Rume le menerent,

e l'apostoilité li donerent.

Hier endet in $B_{1}$ die Legende, und es folgt nur noch ein Schlufs. Somit fehlt die in den andern Handschriften geschilderte und nicht gut zu vermissende Sühnescene der Mutter. Dafs $\mathrm{B}_{2}$ dieselbe hat, ist auffällig und führt, da die Handschrift sich als Mischhandschrift erweist, zu dem Verdachte, dafs diese Scene aus A geschöpft sei. Dieser Verdacht findet sich bestätigt. Stammte diese Scene in $B_{2}$ aus einer B-Redaktion, so mü/ste sich darin die Eigentümlichkeit kund thun, welche die Fassung von B im Gegensatz zur originalen A zeigt, nämlich, dafs sich sichtliche Kürzungen des Textes darbieten. Ist sie nicht original, und stammt sie aus $A$, so muls sie sich in ihrer Lesart an diejenige Handschrift anlegen, die $B_{2}$ neben einer B-Fassung benutzte.

Nun lassen sich keinerlei Kürzungen auffinden; nur einige Verse fehlen, die aber aus Versehen ausgefallen sein können. Dafür aber lehnt sich $B_{2}$ in dieser Scene genau an $A_{2}$ an, wie in den Partien, wo $\mathrm{B}_{2}$ deutlich sich als Mischhandschrift kund giebt, worüber wir uns in $\S 5$ näher verbreiten werden. Daraus folgt, dafs die Sühnescene, wie sie $B_{2}$ aufweist, nicht in einer originalen $\mathrm{B}-$ Redaktion vorhanden gewesen ist, also vermutlich schon in der Vorlage von $\mathrm{B}_{2}$ gefehlt hat. $\mathrm{Da}$ aber auch $\mathrm{B}_{1}$ sie nicht hat, ist es wahrscheinlich, dafs schon die gemeinschaftliche Vorlage von $B_{1} B_{2}$ sie nicht besals. Doch damit ist noch nicht gesagt, dafs sie der B-Redaktion überhaupt fremd gewesen wäre. Vielmehr scheint mir, als ob die letzten Folio-Seiten der gemeinschaftlichen Vorlage durch irgend einen Zufall zerstört worden seien. Dafür sprechen einmal der in $B_{1}$ ganz von den A-Handschriften abweichende Schlufs, während doch in der Einleitung $A$ und $B$ zusammen gehen, anderseits aber auch der Umstand, dafs $B_{1}$ und die anderen Handschriften in einem Punkte sich geradezu widersprechen. Nach den Handschriften $A_{1} A_{2} A_{3} B_{2}$ ist Gregor entweder uns de ceauz oder celui qui chant trova, in $\mathrm{B}_{1}$ heilst es aber: 
Ceo ne fud cil Gregoires mie qui fist les livres e les chanz, ainz fud un altre si vaillanz.

Daraus möchte ich schliefsen: In der Vorlage von $B_{1} B_{2}$ waren Sühnescene und Schluls zerstört, $B_{2}$ schöpfte beides aus $A ; B_{1}$ fügte an das ihr in der Vorlage Gegebene einen eigenen Schlufs. Ja es scheint mir sogar wahrscheinlich, dafs die Schlufsverse in der Vorlage von $B_{1} B_{2}$ die oben genannten gewesen wären:

Es vus les clercs joians e liez, ignelement saillent en piez,

worauf $B_{2}$ das Fehlende aus $A$ ergänzte, $B_{1}$ aber vor der Hinzufügung des Schlusses auch die 2 die Papstwahl Gregor's uns mitteilenden Verse:

Tut dreit a Rume le menerent.

e l'apostoilité li donerent,

eigenmächtig geschaffen hätte.

§ 4. Verwandschaft der A-Handschriften unter einander.

Gemeinsame Fehler in $A_{2} A_{3}$ beweisen, dafs diese Handschriften näher unter sich verwandt sind, als mit $A_{1}$.

Ich führe folgende Beispiele an:

I. 48,3-4 liest $\mathrm{A}_{1}$ :

amis, dist il, grammaire ses,

e des lettres es doctrinés,

während $\mathrm{A}_{2} \mathrm{~A}_{3}$ für grammaire : granment resp. grandement schreiben.

2. $50,1-5$ liest $A_{1}$ :

sire, je trois ici escrit, si c'est veirs que la letre dit, d'un enfant enci faire chose merveille est com om faire l'ose.

Für faire l'ose schreiben $\mathrm{A}_{2} \mathrm{~A}_{3}$ dire l'ose, also sah der Schreiber ihrer gemeinschaftlichen Vorlage in faire das Synonymum von dire, während es hier in seiner gewöhnlichen Bedeutung steht.

3. 53,23-54, 1 liest $A_{1}$ :

de guerre avons sovent tiel fais

que estre ne poons en pais

Tos nos a a povreté trais.

$\mathrm{A}_{2} \mathrm{~A}_{3}$ lassen in $54, \mathrm{I}$ ein $a$ aus.

4. $66, \mathrm{I} I-\mathrm{I}_{2}$ liest $\mathrm{A}_{1}$ :

lores fu deables angoisos,

quant ce oï, e molt joios.

Die Vorlage von $A_{2} A_{3}$ zeigt Abneigung gegen künstliche Wortstellungen und ersetzt sie gern durch natürliche. Dehalb finden wir in $\mathrm{A}_{2} \mathrm{~A}_{3}$ für e molt joios: molt fu joios, wodurch fälschlich der 
Satz: quant ce ö̈ zu nur 66,1 2 gezogen wird, während er doh notwendig auch zu 66, I I gehören muls.

5. $67,3-4$ liest $A_{1}$ :

quant li dux fu devant la dame, il ot el cuer d'amor la flame.

$\mathrm{A}_{2} \mathrm{~A}_{3}$ schreiben für cuer: cors.

6. 82,9-16 liest $A_{1}$ :

tant avons fait que ne crei mie

que ja s'ert clerz qui ce nos die

qui ja poissons espeneir,

ne la penitence sofrir

del pechié dont somes colpable

par la poissance del deable,

se nos ensi fait l'ëusson

qu'a escient le feïsson.

Für feïsson las die Vorlage von $\mathrm{A}_{2} \mathrm{~A}_{3}$, wohl beeinflufst durch das vorhergehende escïent, sëussons. Dadurch entstand eine Tautologie mit Sinnesstörung, die auch $A_{2}$ noch aufweist. Der Schreiber von $\mathrm{A}_{3}$ bemerkte den Fehler und änderte qu'a escïent zu que devant ce.

7. Der Fischer verspricht Gregor, ihn zum Felsen bringen zu wollen und ruft nach $A_{1} 94,5-6$ aus:

Se dex me volt ma nef garir,

de mon cors ne poet faillir.

Sinn ist: Wenn Gott nur mein Schiff bewahrt, an mir soll es nicht fehlen. Die Vorlage von $\mathrm{A}_{2} \mathrm{~A}_{3}$ hatte fälschlich ma nef und mon cors vertauscht, wodurch den Sinn entstellt wird. Deshalb änderte $\mathrm{A}_{3}$ nachträglich $94,6 \mathrm{zu}$ :

et ma nef, n'y porrez faillir,

machte also sowohl ma nef als mon cors abgängig von volt garir.

8. $42,5-1$ I lauten in $A_{1}$ :

li pescheres qui povres fu,

qui les dix mars aveit ëu,

sa feme l'ot tant angoissé,

un jor en autre enuié

qu'il li deïst ou il trova

les äix mars d'argent, qu'il conta

coment Gregoires fu trovés.

Das qu'il conta 42, 10 (aufzufassen als: s'il conta = und er erzählte) hatte der Schreiber der Vorlage von $\mathrm{A}_{2} \mathrm{~A}_{3}$ nicht verstanden und geändert zu: que li dona. Dann mülste doch aber notwendig ein et vor coment 42, I I treten, da dieser Vers auch abhängig von dëist 42,9 wird.

9. 47,9-I 2 liest $A_{1}$ :

et des or m'a en veir promis

que a nuil ome que seit vis 
par jue ne par rien que il face,

n'iert mais sëu en nule place.

Für jue 47, I I lesen $A_{2} A_{3}$ luz, was keinen Sinn giebt.

10. $48,1-2$ lauten in $A_{1}$ :

car tout ai en chevalerie

e mon cuer torné e ma vie.

$\mathrm{A}_{2} \mathrm{~A}_{3}$ fehlt $e$ am Anfange 48,2. Um den Vers metrisch richtig zu stellen, wurde von $\mathrm{A}_{2}$ torné zu atorné, von $\mathrm{A}_{3}$ e ma vie zu toute má vie geändert. Daneben aber lassen beide Handschriften cors für cuer treten, wodurch der Sinn entstellt wird.

I I. p. 78,12-I4 liest $\mathrm{A}_{1}$ :

s'il ot aprés ou mal ou bien,

s'il vesqui a duel ou a honte,

s'il morut, je n'en tin conte.

$\mathrm{A}_{2} \mathrm{~A}_{3}$ fehlt aprés. Dadurch wurde v. I 2 sechssilbig. Diesen Fehler suchten beide Handschriften auszumerzen. Sie schreiben für s'il : se il. Dadurch wurde der Vers immer erst 7-silbig, denn wenn sie auch ot $\mathrm{zu}$ eust und demgemäls vesquit und morut $\mathrm{zu}$ vesquist und morust änderten, so mufs man eust doch einsilbig auffassen, da es hier nicht Subjunktiv sein kann.

I 2. Nachdem Gregor seiner Mutter gesagt hat, wie sie Bufse thun soll, fährt er $A_{1}(84,11-12)$ fort:

je menrei autresi mon cors, si m'en irai del regne hors.

$\mathrm{A}_{2} \mathrm{~A}_{3}$ haben für menrai : metrai.

Diese Resultate werden besätigt durch $\mathrm{A}_{2} \mathrm{~A}_{3}$ allein gemeinsame Zusätze und Auslassungen.

I. 20,17-22 liest $A_{1}$ :

quant la dame li ot ce dire,

cuida que le vosist ocire.

„Dame“, fait el, „por deu le grant,

mi sire par est leaus tant

que ja n'iert conte ne plait

que omecides par lui seit falt."

Wie hier, leitet $A_{1}$ auch sonst direkte Reden gern direkt ein. Dem Schreiber der Vorlage von $\mathrm{A}_{2} \mathrm{~A}_{3}$ und in noch grölserem Mafse dem von $A_{2}$ gefiel dies ebensowenig, wie die in $A_{1}$ häufig vorkommenden künstlichen Wortstellungen und Enjambements. So wird auch hier die direkte Rede indirekt eingeleitet, indem für 20,17-1 8 vier Verse gesetzt und v. 19--20 geändert werden, da fait el in 19 wegen dieser Änderung überflüssig geworden ist. So lautet die Stelle z. B. in $\mathrm{A}_{2}$ :

quant la dame li oï dire, si ot al cuer dolor et ire, cuida que mordrir le volsist, parla apres, et si li dist:

Zeitschr. f. roin Phil. $\mathbf{X}$. 
France dame, por dieu nel dire, car tant loiax par est li sire etc.

2. $21,7-12$ liest $A_{1}$ :

cil vint a li por conforter, mais n'i pot nul confort trover.

Grant ire e grant dolor demeine, de meinte maniere se peine.

Assez prient, mais poi lur vaut, car de priere ne li chaut.

Ganz ohne Not schieben $A_{2} A_{3}$ hinter 2 I, 10 zwei Verse ein:

savoir se ja porroit retraire son pensé de folie faire.

Wird hier der Einschab eingeleitet durch ein savoir, so auch 3. hinter 108,2.

$\mathrm{A}_{1}$ liest $108,1-2$ :

Gregoire les rova aler

la o sis liz fu, regarder.

Hierhinter haben $A_{2} A_{3} 4$ Verse, deren Fehlen in $A_{1}$ das Verständnis durchaus nicht stört:

savoir se ja fussent trovees

la ou il les ot obliees,

et li ostes les i mena,

le lit Gregoire lor mostra.

4. $4 \mathrm{I}, 16-20$ lauten in $\mathrm{A}_{1}$ :

de lui dient petit e grant

que molt iert ja bel enfant; onques mais fils a pescheor ne nasqui de si grant valor.

Auf diese Verse lassen $A_{2} A_{3}$ ganz unnötig 2 Verse folgen:

cascun cuidoit qui le veoit

qu'il fust ses fiex; mais non estoit.

5. Nicht nur nicht überflüssig, sondern sogar störend sind die Verse, welche $A_{2} A_{3}$ hinter 1 i 8,2 einschieben:

nos laist iceles oeuvres faire

que a bone fin puisson traire.

6. Unursprünglich und unnötig ist endlich die Zerdehnung des v. 97,8 :

si parlerons de l'A postoile

zu :

si parlerons d'un Apostoile qui a cel temps estoit a Rome.

Molt le tenoient a proudome,

wie $A_{2} A_{3}$ lesen. 
Von den Zusätzen wollen wir uns zu den $A_{2}, A_{3}$ gemeinsamen Auslassungen wenden.

I. $88,22-24$ liest $A_{1}$ :

une bele toaille prist, si l'estendi en un bel lue

bien pres de lui e pres del fue.

Die Verse 88,23-24, die durchaus nötig sind, fehlen $A_{2} A_{3}$. $\mathrm{A}_{3}$ bemerkte den Fehler und änderte v. $22 \mathrm{zu}$ :

une nappe devant li mist.

2. Alsdann fehlen die Verse 30,21-22, deren Nichtvorhandensein allerdings nur dann beweisend für die Zusammengehörigkeit von $A_{2} A_{3}$ sind, wenn sie ursprünglich, nicht etwa Einschub von $A_{1}$ sind.

3. Endlich fehlen $A_{2} A_{3}$ die Verse 50,21-22. Allerdings scheinen sie in $A_{1}$ die Konstruktion zu stören, doch sie stehen an falscher Stelle. Sie müssen vor 50,18-19 gestellt werden. Dann ist auch der Ausfall sehr leicht erklärlich, da 50,1 8 und 50,20 den Versanfang et puis hatten.

Unseren Resultaten scheinen nur zwei Fälle zu widersprechen:

I. die gemeinschaftliche Übereinstimmung von $A_{1} A_{2}$ I05, I I: n'aveit fors le cuer e les os,

wo cuer für cuir steht. Allein hier haben wir es mit einem Schreibfehler zu thun, den zwei Schreiber, unabhängig von einander, sich zu Schulden kommen liefsen.

2. die gemeinschaftliche Übereinstimmung von $A_{1} A_{3}$ 20,6: dame, fait il, un fiz avez,

wo il für $e l=$ illa steht. - Doch, wie wir Teil I, $\S$ I gesehen haben, sind die Fälle zahlreich, in denen $A_{1}$, weil von einem provenzalischen Schreiber herrührend, il und el vertauscht.

\section{$\S$ 5. Verwandtschaft der B-Handschriften mit den A-Hand- schriften.}

I. Die Vorlage von $B_{1} B_{2}$ ging auf eine A-Handschrift zurück, welche der durch $A_{2} A_{3}$ vertretenen Gruppe angehörte.

Dies beweisen gemeinschaftliche Fehler in $A_{2} A_{3} B_{1} B_{2}$.

I. $20, \mathrm{I}-4$ liest $\mathrm{A}_{1}$ :

la chambre fu molt serree ou la dame s'est delivree, onques n'i ot autre al veiller fors que la dame el chevaler.

Für autre al veiller schreiben die 4 anderen Handschriften: au travailler. 
2. Gregor's Mutter fragt den Boten, der ihr den Tod ihres Bruders anzeigt, wann und auf welche Weise er krank wurde, und an welchem Tage er starb. Der Bote antwortet ( $\left.A_{1} 29,1_{2}-I_{5}\right)$ :

$$
\begin{aligned}
& \text { dame, fait il, en icele ore } \\
& \text { que tu de lui te departis, } \\
& \text { lui prist li maus qui l'a ocis, } \\
& \text { e mors fu a une jornée. }
\end{aligned}
$$
l'a ocis.

$\mathrm{A}_{2} \mathrm{~A}_{3} \mathrm{~B}_{1} \mathrm{~B}_{2}$ lesen für: li mais qui l'a ocis : la mort qui

3. Als das ausgesetzte Kind auf dem Meere umhertreibt, treffen dasselbe zwei Fischer von denen es $\left(A_{1} 34,7-I C\right)$ heifst:

li abes meismes sis cors

les ot la nuit enveié hors

por peisson prendre en cele mer,

a tos ses moines conreer.

Sinn ist: Der Abt hat die Fischer zum Fischfang ausgesandt, damit er mit der gewonnenen Beute seine Mönche versorgen könnte. Für a tos 34 , 10 schreiben die 4 anderen Handschriften ganz unsinnig avec.

4. $23,15-20$ lauten in $A_{1}$ :

ce chatel doins a l'enfant:

l'or et le paile reluisant.

Les tables gart qui sont d'ivoire

ou est escrit de lui l'estoire,

por deu le grant itant de tens

que apris ait auques de sens.

Der Schreiber der gemeinschaftlichen Vorlage von $A_{2} A_{3} B_{1} B_{2}$ glaubte, die Worte les tables mülsten auch abhängig sein von doins. Deshalb liefs er in v. 17 gart aus und schrieb für les tables : et les tables. Ohne Berechtigung und ohne Verbindung steht somit itant de tens in v. 20. Diese erste fehlerhafte Lesart zeigt auch noch $\mathrm{A}_{2}$. Die anderen Handschriften merzten unabhängig von einander diesen Fehler aus. $\mathrm{A}_{3}$ änderte deu le grand in $\mathrm{v} .19 \mathrm{zu}$ deu le garde. $B_{1} B_{2}$ stellen $v .15$ und 16 um und lesen:

e le chier paile e l'or luisant

duinst a chatel a cel enfant,

ändern aber aufserdem $v_{-} 19$ und $20 \mathrm{zu}$ :

tres bien les gart pur deu le grant,

e puis si rende a cel enfant.

5. $14,10-12$ liest $A_{1}$ :

quant il descendi as degrés,

e li vaslés venir le vit,

entre ses bras le recoillit.

Den Reim dit: recoillit ändern die andern Handschriften zu voit : regoit. Dadurch wurde v. 12 siebensilbig, wie ihn $A_{2} B_{1}$ auch 
noch haben. $B_{2} A_{3}$ merzten diesen Fehler aus, indem $B_{2}$ bel vor le setzte, $A_{3}$ deux zwischen ses bras einschob.

6. 74, 19-22 lauten in $A_{1}$ :

les joies durent longement

l'accler e l'cmbracement

que la mere vers son fiz meine

de ci qu'a none tote pleine.

Wir finden in den ersten beiden Versen wieder eine künstliche Wortstellung. Diese behagte dem Schreiber der Vorlage der anderen Handschriften nicht. Deshalb zerdehnte er diese 2 Verse zu 4:
sos ciel n'a clerc qui pëust dire,
qui tant sëust cunter ne lire
les joies, les embracemens,
l'acoler et les baisemens.

Im Anschlufs hieran führe ich ein Beispiel an, welches beweist, dafs der Schreiber der Vorlage von $A_{2} A_{3} B_{1} B_{2}$ auch kein Enjambement litt. 9,19-20 liest $\mathrm{A}_{1}$ :

quar, vueile ou non, l'a violee

sis freres, e depucelee.

Dafür die andern Handschriften:

ou vueille ou non la bele nee,

si l'ad sis freres violee.

7. Daneben zeigt sich die Eigentümlichkeit, dafs die ursprüngliche Vorlage von $A_{2} A_{3} B_{1} B_{2}$ Übergänge mit Hülfe von Zeitadverbien nicht für genügend erachtete. So liest $A_{1}$ 49,2 I-23:

cil a fait ce qu'il li rova,

les letres list que il trova.

Lors a son parein regardé.

Die andern Handschriften verstärken den durch lors angezeigten Übergang, indem sie in Anschlufs an 37,17-18 zwischen 49,22 und 23 zwei Verse einschieben:

et quant il ot les letres lites qui es tables furent escrites,

nur hat vor diesen $B_{2}$ noch 4 andere:

que qu'il les list, souspire et pleure;

li bons abes prie et aeure que damediex le convertisse si ke l'aglyse ne guerpisse.

8. $54,21-22$ liest $A_{1}$ :

e se madame vos veeit, molt volentiers vos retendreit.

Hierhinter haben $A_{2} A_{3} B_{1} B_{2}$ zwei Verse, von denen es fraglich ist, ob sie original, oder nur Einschub sind. Ich citiere nach $\mathrm{A}_{2}$ : 
car molt samblés gentil baron al vis, al cors, a le façon.

Sind sie nicht original, so sind sie ein weiterer Beweis für die Zusammengehörigkeit der 4 Handschriften.

II. Die Vorlage von $B_{1} B_{2}$ war näher mit $A_{2}$ als mit $A_{3}$ verwandt.

$\mathrm{Ob}$ nun eine $\mathrm{A}_{2}$ - oder eine $\mathrm{A}_{3}$-Handschrift die Vorlage der Handschrift war, aus der $B_{1} B_{2}$ entstammten, diese Frage ist schwer zu beantworten, da den Beweisen, die für $A_{2}$ sprechen, doch nicht ganz ungewichtige entgegen stehen, die $B_{1} B_{2}$ näher mit $A_{3}$ verwandt erscheinen lassen. Allein auf Grund viel: $\mathbf{r}$ ganz auffällig mit einander übereinstimmenden Lesarten und einiger gemeinsamen Fehler wage ich $A_{2} B_{1} B_{2}$ zusammen zu stellen.

I. Als der Abt die Tonne sieht, in der das Kind ruht, fragt er die beiden Fischer, was in derselben enthalten sei. Sie antworten nach $A_{1} A_{3} j 6,5-6$ :

Il li ont dit: de nos afaires,

sire, n'i ad de chose guaires.

Sinn ist: Von dem was wir bringen sollten, giebt es darin nichts. $\mathrm{A}_{2} \mathrm{~B}_{1} \mathrm{~B}_{2}$ schreiben ganz sinnlos für de nos afaires: cest nos afaires.

2. 20,19-20 lesen $A_{1} A_{3}$ :

e tant en fu sis cors pensis

qu'onques n'i ot ne joi ne ris.

$\mathrm{A}_{2} \mathrm{~B}_{1} \mathrm{~B}_{2}$ schreiben für cors : cuers.

3. 75, $15-20$ lauten in $A_{1} A_{3}$ :

ceste enfertez est si averse,

si honie e si desperse

qu'en terre n'a cele racine

qui me pöust faire mecine,

ne ja n'en avrai garison

ne par herbe, ne par poisson.

$\mathrm{A}_{2} \mathrm{~B}_{1} \mathrm{~B}_{2}$ haben für herbe : mire. $\mathrm{B}_{2}$ schreibt zwar mie, doch man sieht leicht, dafs dies ein blofser Schreibfehler für mire ist.

4. Beweisend scheint mir auch der Zusatz zu sein, den $A_{2}$ $B_{1} B_{2}$ allein hinter 17,2 haben:

et a tel home commander

que bien le face honorer

de ci a tant que revenras

de cel voiage ou tu iras.

In den Handschriften $A_{1} A_{3}$ fehlen diese Verse eben so gut, ohne den Zusammenhang zu stören.

$\mathrm{Zu}$ diesen Beispielen treten noch 2, wo zwar nur je eine BHandschrift-mit $A_{2}$ zusammengeht, wo es aber wahrscheinlicher ist, dafs eine B-Handschrift gebessert, als dals die andere, unabhängig 
von $\mathrm{A}_{2}$, mit dieser Handschrift zufällig denselben Fehler sich habe zu Schulden kommen lassen.

5. 45, 15-16 liest nämlich $A_{1}$ : amis, dist l'abes, atendés tant que les aie araisonés.

Für atendés schreiben $A_{2} B_{1}$ fälschlich: entendez.

6. $37, \mathrm{I}$ I liest $\mathrm{A}_{1}$ :

embedeus mains vers deu etent;

$A_{2} B_{2}$ aber schreiben fälschlich entent für etent.

Diesem Resultate scheinen 3 Fälle zu widersprechen und $z$ war sind es gemeinsame Zusätze in $A_{3} B_{1} B_{2}$. Dieselben zwingen nicht zu der Annahme, dafs sie original wären; soll unsere Hypothese aber richtig sein, so müssen dieselben auch in der Vorlage von $\mathrm{A}_{2}$ gestanden haben.

I. $19,16-18$ lauten in $A_{1}$ :

e li promet tres bien e jure

que ja de rien n'iert descoverte

por nul grant gaaing ne por perte.

Hierhinter haben $A_{3} B_{1} B_{2}$ zwei Verse:

I8a ne ya de rien n'yert tant iree

I8b que ya en soit depoepliee.

Der Ausfall dieser Verse in $A_{2}$ ist jedoch sehr leicht erklärlich, da v. I 7 und 18 a zur grölsten Hälfte gleichlauten:

2. Einen weiteren Zusatz haben $A_{3} B_{1} B_{2}$ hinter 52,6:

$6 a$ et chascun jor dieu en deprie

$6 \mathrm{~b}$ que droite voie le conduie.

Nun beginnt aber 52,5 in $A_{2}$ mit et, ebenso auch 52,6a; deshalb ist es nicht unwahrscheinlich, dafs dieser gleiche Versanfang den Ausfall der Verse 52,6a und b veranlafst habe.

3. $69,1-2$ lauten in $A_{1}$ :

tant s'est deables entremis

que la mere a son enfant pris.

Diese Verse fehlen $B_{1}$, dafür hat die Handschrift 2 Verse, die $\mathrm{B}_{2} \mathrm{~A}_{3}$ nach diesen Versen aufweisen. So liest $\mathrm{A}_{3}$ :

2a qui oncques hommes ne fina

2b jusques a ce qu'ou lict les yousta,

wofür sich in $B_{1} B_{2}$ vier Verse finden:

2a tant que li diable ne finat

2b de si qu'al lit les ajosta.

2c Il fait l'un l'altre aveir mult chier

2d pur le pechié bien aducier.

Dafs die beiden Verse, welche $A_{3} B_{1} B_{2}$ aufweisen, in der Vorlage von $A_{2}$ gefehlt hätten, ist um so unwahrscheinlicher als $\mathrm{B}_{2}$ sich hier wieder als Mischhandschrift kundgiebt, in solchen Partien sich sonst aber ganz ohne Frage an $A_{2}$ anlehnt. Wie ist 
nun aber der Ausfall zu erklären? Von Einflufs könnte allerdings deable in 69, 1 und 69,2a gewesen sein. Allein ein anderer Grund ist mir einleuchtender. Wie wir an einer anderen Stelle gesehen haben, liebt $\mathrm{A}_{2}$ die Anaphora. Nichts scheint mir natürlicher als dals eine solche eine frühere $A_{2}$-Handschrift an dieser Stelle gehabt, also v. 69,2a vielleicht gelesen hätte: tant li diables ne fina. Da lag die Gefahr sehr nahe, dals durch Abirren des Auges die beiden Verse verloren gingen. - Auf das gemeinschaftliche Fehlen der Verse 39,13-I4 und 40,9-IO in $A_{3} B_{1} B_{2}$, sowie auf das der Verse $42, I_{1} d$ und 12 in $A_{2} B_{1} B_{2}$ ist kein Gewicht zu legen, da die B-Handschrift prinzipiell Verse auslassen, welche ohne Störung übergangen werden können, in $A_{2}$ oder $A_{3}$ die betreffenden Verse aber aus Zufall übersehen sein könnten.

\section{III. $B_{2}$ hatte neben einer $B$ - eine $A_{2}$-Vorlage.}

Ist die Abstammung der Vorlage von $B_{1} B_{2}$ aus einer $A_{2}$ Handschrift wenig sicher, so zeigt es sich ganz deutlich, dals in den Partien, wo $B_{2}$ sich als Mischhandschrift erweist, eine $A_{2}-$ Handschrift benutzt wurde.

Zunächst sind einige Fälle zu nennen, aus denen es sich ergiebt, dafs $B_{2}$ eine A-Handschrift zur Vorlage hatte, die der durch $\mathrm{A}_{2} \mathrm{~A}_{3}$ vertetenen Gruppe näher stand, als $A_{1}$.

I. 64,9-I I liest $A_{1}$ :

a dreite force, a mal talent, molt les menoient malement.

Toz les destrenchoent a fais.

Für a fais schreiben $\mathrm{A}_{2} \mathrm{~A}_{3} \mathrm{~B}_{2}$ fälschlich $a$ fait und ändern demgemäls plais in 64,12 zu plait.

2. Hinter 61,2 schieben $A_{2} A_{3} B_{2}$ zwei Verse ein, die wegen der Verse 6I,4-5 unnötig sind:

porpense soi c'a lui ira,

et encontre lui jostera.

Zahlreicher sind die Fälle, wo $A_{2}$ allein mit $B_{2}$ zusammen geht.

I. 89,20-22 liest $A_{1}$ :

Tu manjeroies tot le peisson

de chief en chief jusqu'en l'areste

par les dous oilz de ceste teste. la teste.

Für l'areste in $89,2 \mathrm{I}$ schreiben $\mathrm{A}_{2} \mathrm{~B}_{2}$, durch $8,9,22$ beeinflufst,

2. Hinter 63,20 haben $A_{2} B_{2}$ zwei Verse:

vers lui poignerent qui ains ains,

grant honte avint cel jor as mains.

Beeinflufst ist dieser Einschub dadurch, dafs der Schreiber ihrer Vorlage nicht bemerkt hatte, dafs v. 21-22 in Parenthese zu 
schliefsen seien. Er glaubte v. I9 fehlte der Hauptsatz und schob deshalb 2 Verse ein, während der Hauptsatz in v. 23 und $24 \mathrm{zu}$ suchen ist. Absichtlich liefs später ein Abschreiber von $A_{2}$ v. 23 und 24 aus, während $B_{2}$ diese Verse noch aufweist.

3. Hinter I I 3, I 2 haben $A_{2} B_{2}$ einen Zusatz von 2 Versen:

a dame deu graces en rent, si li conforte sagement.

Grund ist: Herstellung eines Überganges zur direkten Rede. 4. Einen unnötigen Zusatz haben ferner $\mathrm{A}_{2} \mathrm{~B}_{2}$ hinter I I 4,2: car ore est plus bone eüree que nule femme qui soit nee.

5. Endlich zeigen $A_{2} B_{2}$ einen grölseren Einschub hinter 1 1 4, 18 :

puis se porpense en son corage,

et dist: or ne sui jo pas sage;

mort desirier est grant folie, miex doi jo desirier la vie, et travaillier et moi pener que a deu me puisse acorder.

Den letzten Vers zerdehnt $B_{2} z u$ dreien:

que coroune puisse acater o cheus ke diex velt coroner, et en se gloire o lui poser,

Diese Verse sind veranla/st durch I 14,2 I-22: cil esteit liez, e deu loot qui a bien faire la tornot.

Doch ist es nicht zwingend, die in $A_{2} B_{2}$ allein enthaltenen Verse für original zu halten. Die Freude Gregor's über die Bekehrung seiner Mutter zum Guten kann man darauf beziehen, dafs sie überhaupt nach Rom gekommen ist, um Bufse zu thun.

6. Gemeinschaftlich sind dann noch $A_{2} B_{2}$ die Lücken I I5, 9-10, 84,17-18.

Diesem Resultate scheinen 2 Fälle zu widersprechen. Verse:

I. das Fehlen der in $A_{2} A_{3}$ hinter 118,2 eingeschobenen

nos laist iceles oeuvres faire que a bone fin puisson traire.

Allein vielleicht hat der Schreiber sie absichtlich unterdrückt, da sie an der Stelle störend wirken.

2. Auffällig aber bleibt das Vorhandensein der Verse 118 , 5-6, die allein in $A_{1} B_{2}$ überliefert werden, während $A_{2} A_{3}$ die Legende mit I 8,4 schliefsen. Sollte Luzarche sich etwa auch hier wieder in den Zahlen geirrt und 8 statt 6 Verse am Schlusse $\mathrm{B}_{2}$ entlehnt haben? 
362 w. MIEHLE, DAS VERHÄLTNIS DER HSS. DES ALTFRZ. GREGORIUS.

\section{Schlufs.}

Fassen wir das Resultat unserer Untersuchung zusammen, so ist es folgendes:

Es liegen uns von der Legende zwei von einander stark abweichende Redaktionen vor, eine A- und eine B-Redaktion. Von den Handschriften der A-Redaktion sind $A_{2} A_{3}$. näher unter sich verwandt, als mit $A_{1}$. Die ursprüngliche $B$-Redaktion ging auf eine $\mathrm{A}_{2}$-Handschrift zurück, während die $\mathrm{B}_{2}$-Handschrift neben einer $\mathrm{B}$-Vorlage eine $\mathrm{A}_{2}$-Handschrift benutzte.

Nennen wir die Originalhandschrift $\mathrm{O}$, die gemeinschaftliche Quelle der vorhandenen fünf Handschriften $x$, die Vorlage von $A_{2} A_{3}: A_{x}$, die von $B_{1} B_{2}: B$, so stellt sich die Klassifikation der Handschriften folgendermalsen:

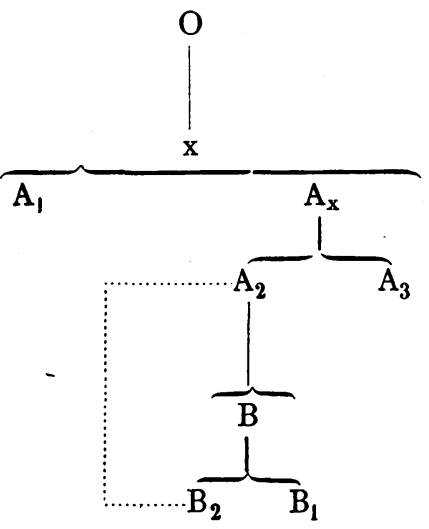

W. Miehle. 